\title{
Genomic encyclopedia of sugar utilization pathways in the Shewanella genus
}

\author{
Dmitry A Rodionov ${ }^{1,2}$, Chen Yang ${ }^{1,3}$, Xiaoging Li ${ }^{1}$, Irina A Rodionova ${ }^{1}$, Yanbing Wang ${ }^{4}$, Anna Y Obraztsova ${ }^{4,7}$, \\ Olga P Zagnitko ${ }^{5}$, Ross Overbeek ${ }^{5}$, Margaret F Romine ${ }^{6}$, Samantha Reed ${ }^{6}$, James K Fredrickson ${ }^{6}$, \\ Kenneth $\mathrm{H}$ Nealson ${ }^{4,7}$, Andrei L Osterman ${ }^{1,5^{*}}$
}

\begin{abstract}
Background: Carbohydrates are a primary source of carbon and energy for many bacteria. Accurate projection of known carbohydrate catabolic pathways across diverse bacteria with complete genomes constitutes a substantial challenge due to frequent variations in components of these pathways. To address a practically and fundamentally important challenge of reconstruction of carbohydrate utilization machinery in any microorganism directly from its genomic sequence, we combined a subsystems-based comparative genomic approach with experimental validation of selected bioinformatic predictions by a combination of biochemical, genetic and physiological experiments.
\end{abstract}

Results: We applied this integrated approach to systematically map carbohydrate utilization pathways in 19 genomes from the Shewanella genus. The obtained genomic encyclopedia of sugar utilization includes $\sim 170$ protein families (mostly metabolic enzymes, transporters and transcriptional regulators) spanning 17 distinct pathways with a mosaic distribution across Shewanella species providing insights into their ecophysiology and adaptive evolution. Phenotypic assays revealed a remarkable consistency between predicted and observed phenotype, an ability to utilize an individual sugar as a sole source of carbon and energy, over the entire matrix of tested strains and sugars.

Comparison of the reconstructed catabolic pathways with E. coli identified multiple differences that are manifested at various levels, from the presence or absence of certain sugar catabolic pathways, nonorthologous gene replacements and alternative biochemical routes to a different organization of transcription regulatory networks.

Conclusions: The reconstructed sugar catabolome in Shewanella spp includes 62 novel isofunctional families of enzymes, transporters, and regulators. In addition to improving our knowledge of genomics and functional organization of carbohydrate utilization in Shewanella, this study led to a substantial expansion of our current version of the Genomic Encyclopedia of Carbohydrate Utilization. A systematic and iterative application of this approach to multiple taxonomic groups of bacteria will further enhance it, creating a knowledge base adequate for the efficient analysis of any newly sequenced genome as well as of the emerging metagenomic data.

\section{Background}

Carbohydrates comprise a key natural source of carbon and energy for a variety of heterotrophic microbes. The diversity of carbohydrates (polymers, oligo- and monosaccharides) in various ecosystems as well as the diversity of microbial lifestyles is reflected in substantial variations of sugar catabolic machinery even between

\footnotetext{
* Correspondence: osterman@burnham.org

'Burnham Institute for Medical Research, La Jolla, California 92037, USA

Full list of author information is available at the end of the article
}

phylogenetically closely-related microorganisms. An ability to confidently reconstruct this machinery directly from genomic sequence is crucial to an understanding of microbial ecophysiology, evolution, adaptation, and even interactions between microorganisms and their animal, insect, or plant hosts. However, despite many studies, the bulk of our knowledge about sugar utilization pathways is limited to a handful of model bacteria such as $E$. coli. An accurate projection of this knowledge across the growing variety of divergent bacteria with completely sequenced genomes constitutes a substantial

\section{() Biomed Central}


challenge. This is largely due to the aforementioned variations including numerous cases of non-orthologous gene replacements, families of paralogous proteins with varying substrate specificity as well as alternative and presently unknown biochemical routes. Due to this complexity, genomic annotations of carbohydrate utilization genes derived solely from sequence similarity analysis are often imprecise and incomplete, especially for relatively distant and poorly studied bacteria.

Shewanellaceae are a versatile family of Gram-negative $\gamma$-proteobacteria that have adapted for survival in highly varied aquatic and sedimentary environments that exhibit extremes in salinity, temperature, redox chemistry, and hydrostatic pressure [1]. Although Shewanella can respire with a diverse array of electron acceptors that include organic compounds and metal oxides [2], they were thought to possess a relatively narrow capacity for utilizing electron donors, preferring simple carbon compounds such as formate, lactate, and acetate. The recent results from comparative and functional genomic analysis [3], physiological experiments [4] and microarray analysis [5] demonstrated that $S$. oneidensis MR-1 can use a wide array of complex carbon compounds including nucleotides, amino acids, mono- and polysaccharides.

A subsystems-based approach to genome analysis allows us to substantially improve the accuracy of genomic annotations and to predict functions of previously unknown gene families [6,7]. This approach includes a combination of comparative genomic techniques such as the analysis of conserved operons and regulons, with pathway reconstruction across a large variety of genomes. Recently, we used a subsystemsbased approach to predict and experimentally verify novel pathways of $N$-acetylglucosamine and lactate utilization in Shewanella $[8,9]$. In the current study we have expanded this analysis towards genomic reconstruction of the entire repertoire of carbohydrate utilization pathways in a group of 19 species from the genus Shewanella with completely sequenced genomes.

\section{Results}

Reconstruction of carbohydrate utilization machinery in Shewanella spp

To address the challenge of genomic reconstruction of the carbohydrate utilization machinery we took advantage of several features characteristic of many sugar utilization pathways, such as:

\section{Uniform functional organization}

A typical sugar utilization pathway includes a transport system for sugar uptake and a set of intracellular enzymes that perform biochemical transformations. Although the latter processes vary from sugar to sugar (and, to a degree, from organism to organism), most of them are performed by a rather narrow spectrum of enzymatic activities such as kinases, isomerases, oxidoreductases, hydrolases, and aldolases. Sugar kinases or phosphoenolpyruvate:sugar phosphotransferase systems (PTS) catalyze an essential phosphorylation step (as all intermediates of central carbon pathways to the point of pyruvate are anchored by one or two phosphates). We also included in this analysis associated transcriptional regulators of sugar utilization pathways that mediate specific induction of a catabolic pathway. For utilization of various natural polysaccharides, many pathways are equipped by upstream hydrolytic enzymes producing oligo-, di- or mono-saccharides that are then transported into the cell and further metabolized. These and other upstream and auxiliary (e.g. involved in sensing and chemotaxis) components of the carbohydrate utilization machinery were typically excluded from our analysis, except for those that share genomic context with other sugar utilization components.

\section{Ubiquitousprotein families}

Despite their functional diversity, many characterized components of sugar utilization pathways occur in a limited number of protein families containing multiple paralogs with varying substrate specificities. Representatives of these families can be recognized by homologybased genomic searches, thus providing a source of gene candidates for pathway reconstruction. On the other hand, homology-based methods, taken alone, often fail to reliably identify the exact substrate specificity. This can be accomplished by additional reasoning based on the analysis of functional and genomic context (functional coupling) [10].

\section{Strong functional coupling}

Bacterial genes involved in sugar catabolism are often organized in compact operons and/or regulated by committed transcription factors. Therefore, genome context analysis techniques [6] based on the identification of conserved chromosomal clusters (operons) and shared regulatory sites (regulons) are particularly efficient for accurate functional assignment of previously uncharacterized genes of sugar utilization pathways.

Based on the above considerations we developed the bioinformatic workflow that was applied for the analysis of 19 complete Shewanella genomes (Fig. 1). The key starting point of this workflow was a collection of manually curated subsystems in the SEED genomic database [7] capturing a substantial fraction of known sugar utilization pathways projected across many bacterial species. A compilation of $\sim 480$ groups of isofunctional homologs from this collection (see additional data file 1) was used as a source of queries for homologybased scanning of 19 Shewanella genomes integrated in the SEED database. The underlying assumption was that any sugar utilization pathway, even extremely divergent 


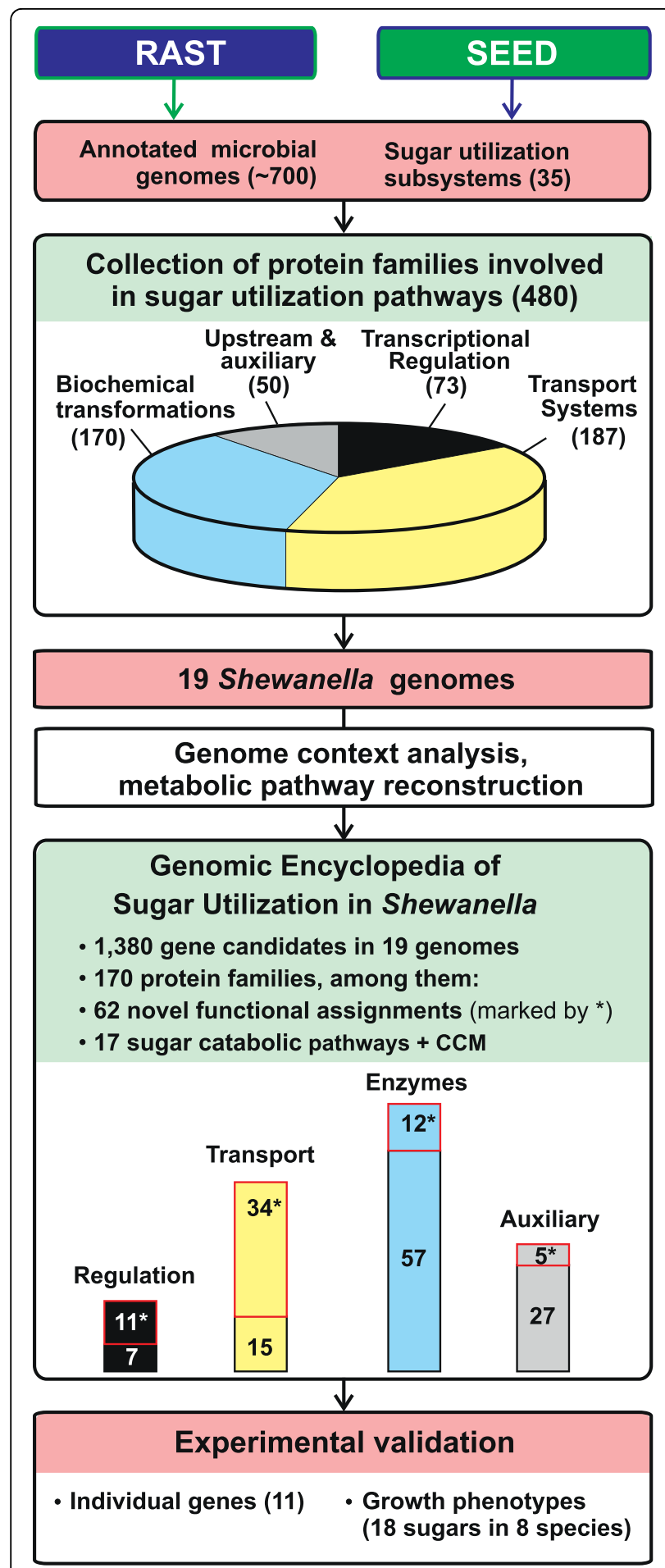

Figure 1 Workflow for genomic reconstruction of carbohydrate utilization machinery in Shewanella spp.

or fully unknown, should contain at least one protein recognizably homologous to some of the query sequences from this collection. Therefore, all of the revealed homologs would be considered potential "kernels" of sugar utilization pathways, even though a specific function of some of them might be unclear (and distinct from the query). During further analysis many of the initially identified gene candidates whose functional roles were deemed unrelated to sugar utilization (e.g. involved in biosynthetic pathways) or remained dubious (lacking any evidence for functional assignment) were rejected.

By applying this workflow we tentatively defined the first draft of the Genomic Encyclopedia of Sugar Utilization (or, shortly, sugar catabolome) in 19 Shewanella spp as comprised of 170 isofunctional protein families (FIGfams) [11]. The complete list and some statistics of the analyzed genomes are provided in Table 1 . The detailed results of this analysis are captured in the SEED subsystem 'Sugar catabolome in Shewanella species' available online http://theseed.uchicago.edu/FIG/subsys. cgi and summarized in additional files 2 and 3. Functional annotation of the identified protein families was conducted using simultaneous genome context analyses, and homology searches combined with the metabolic reconstruction and identification of missing steps in the putative catabolic pathway.

Specific functional assignments have been proposed for 157 FIGfams comprising 17 peripheral sugar utilization pathways and the key components of the central carbon metabolism (CCM) pathways (Table 2, Fig. 2). A total of 21 FIGfams involved in CCM upstream of pyruvate are conserved in all analyzed Shewanella spp. They comprise complete canonical pentose phosphate (PP) and EntnerDoudoroff (ED) pathways. However, an essential enzyme of glycolysis, 6-phosphofructokinase (Pfk), is missing in all of these species suggesting that sugar utilization in Shewanella can proceed only through the ED or PP pathways. This is in agreement with previous metabolic reconstruction reports $[1,3]$ and biochemical studies of CCM enzymes in cell extracts of certain Shewanella strains [12]. All other canonical enzymes of glycolysis/ gluconeogenesis are present in all analyzed Shewanella genomes pointing to the presence of intact gluconeogenetic route. The peripheral pathways are comprised of 136 FIGfams showing a mosaic distribution among 19 compared species. The total number of proteins that comprise the peripheral sugar utilization machinery of individual species varies broadly, from 18 proteins comprising 3 pathways in S. sediminis up to 74 proteins comprising 10 pathways in S. baltica OS223.

13 FIGfams have not been assigned a specific function in any sugar utilization pathway due to the lack of any suggestive genomic context (see additional data files 2 and 3). Among them there are three genes encoding putative sugar kinases of unknown specificity, and genes from a hypothetical sugar utilization gene cluster (for more details see additional data file 4). 
Table 1 Genomic properties and isolation site characteristics of analyzed Shewanella species

\begin{tabular}{|c|c|c|c|c|c|}
\hline Genome & Alias & Total genes $^{1}$ & Sugar genes $^{2}$ & $\begin{array}{l}\text { Sugar } \\
\text { pathways }\end{array}$ & Isolation site characteristics \\
\hline S.oneidensis MR-1 & MR1 & 4318 & 56 & 4 & Lake Oneida, NY, USA (sediment) \\
\hline S.putrefaciens CN-32 & CN32 & 3972 & 64 & 4 & Albuquerque, NM, USA (subsurface) \\
\hline S.putrefaciens W3-18-1 & W3181 & 4044 & 64 & 4 & Washington Coast, Pacific Ocean (sediment) \\
\hline S.sp. ANA-3 & ANA3 & 4111 & 87 & 7 & Eel Pond, Woods Hole, MA, USA (brackish water) \\
\hline S.sp. MR-4 & MR4 & 3924 & 86 & 7 & Black Sea (sea water - 5 m) \\
\hline S.sp. MR-7 & MR7 & 4006 & 92 & 8 & Black Sea (sea water - 60 m) \\
\hline S.baltica OS155 & Sbal & 4307 & 77 & 7 & Baltic Sea (sea water - 90 m) \\
\hline S.baltica OS185 & OS185 & 4323 & 84 & 8 & Baltic Sea (sea water - 120 m) \\
\hline S.baltica OS195 & OS195 & 4499 & 80 & 7 & Baltic Sea (sea water - 140 m) \\
\hline S.baltica OS223 & OS223 & 4250 & 110 & 10 & Baltic Sea (sea water - 120 m) \\
\hline S.denitrificans OS217 & Sden & 3754 & 58 & 4 & Baltic Sea (sea water - 120 m) \\
\hline S.loihica PV-4 & PV4 & 3859 & 59 & 5 & Loihi Seamount, Pacific Ocean (hydrothermal vent) \\
\hline S.amazonensis SB2B & Sama & 3645 & 89 & 6 & Amazon River Delta, Brazil (sediment) \\
\hline S.frigidimarina NCIMB 400 & Sfri & 4029 & 70 & 8 & North Sea, coast of Aberdeen, UK (sea water) \\
\hline S.piezotolerans WP-2 & Spie & 4933 & 68 & 6 & West Pacific site (sediment - 1914 m) \\
\hline S.pealeana ANG-SQ1 & Spea & 4241 & 71 & 6 & Woods Hole harbor, MA, USA (squid gland) \\
\hline S.halifaxens HAW-EB4 & Shal & 4278 & 59 & 5 & Halifax Harbor, Nova Scotia, CA (sediment - 215 m) \\
\hline S.sediminis HAW-EB3 & Ssed & 4497 & 47 & 3 & Halifax Harbor, Nova Scotia, CA (sediment - 215 m) \\
\hline S.woodyi ATCC 51908 & Swoo & 4880 & 84 & 7 & Strait of Gibraltar, Mediterranean Sea (370 m) \\
\hline
\end{tabular}

${ }^{1}$ protein coding genes

2 number of genes involved in the reconstructed sugar utilization pathways.

${ }^{3}$ number of the reconstructed sugar utilization pathways.

Table 2 Reconstructed sugar utilization pathways and newly assigned genes in Shewanella spp

\begin{tabular}{|c|c|c|c|c|c|c|c|}
\hline \multicolumn{2}{|c|}{$\begin{array}{l}\text { Sugar utilization pathway } \\
\text { (subsystem) }\end{array}$} & \multirow[t]{2}{*}{$\begin{array}{l}\text { Number of } \\
\text { genomes }\end{array}$} & \multirow[t]{2}{*}{$\begin{array}{l}\text { Number of } \\
\text { genes }\end{array}$} & \multicolumn{4}{|c|}{ Newly assigned genes } \\
\hline Carbohydrates & Abbrev. & & & regulation & transport & enzymes & auxiliary \\
\hline $\begin{array}{l}\text { Central carbon } \\
\text { metabolism }\end{array}$ & CCM & 19 & 21 & - & - & - & - \\
\hline $\begin{array}{l}N \text {-acetylglucosamine, } \\
\text { chitin }\end{array}$ & Nag & 18 & 15 & $\operatorname{nag} R$ & $\underline{\operatorname{nagP}}$, omp Nag & $\underline{n a g B} B^{\prime \prime}, n a g K$, & $\begin{array}{l}\operatorname{mcp}^{N a g} \\
\operatorname{nag} X\end{array}$ \\
\hline Glycerate & Grt & 17 & 3 & - & $\underline{\text { grtP }}$ & - & - \\
\hline$\beta$-glucosides, cellobiose & $\mathrm{Bgl}$ & 9 & 8 & $b g / R$ & $\underline{\mathbf{b g} / \boldsymbol{T}}, g l c P^{B g l} \circ m p^{B g l}$ & $\underline{\underline{g} \mathbf{k}^{\prime \prime}}$ & - \\
\hline Sucrose & $\mathrm{Scr}$ & 8 & 5 & $s c r R^{\|}$ & $\overline{\text { scrT }}=, o m p^{\mathrm{Scr}}$ & - & - \\
\hline Maltodextrins & Mal & 14 & 13 & malR & 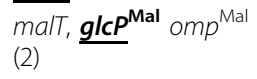 & - & - \\
\hline Arabinose, arabinosides & Ara & 6 & 18 & $\operatorname{araR} \|$ & $\begin{array}{l}\underset{\text { omp }}{\operatorname{ara}} \\
\text { Ara }\end{array}$ & $\operatorname{aram}, \operatorname{araY}$ & $\operatorname{arax}$ \\
\hline Galactose, galactosides & Gal & 8 & 10 & - & galp ${ }^{\|_{1}} o m p^{\text {Gal }}$ & - & - \\
\hline Gluconate & Gnt & 4 & 3 & - & - & - & - \\
\hline $\mathrm{N}$-acetylgalactosamine & Aga & 4 & 8 & - & agaP, omp $p^{\text {Aga }}$ & $\begin{array}{l}\text { agaA", agak, } \\
\text { agas }\end{array}$ & agaO \\
\hline Mannosides & Man & 4 & 14 & $\begin{array}{l}\operatorname{man} R_{1}^{\prime} \\
\operatorname{man} R^{\prime \prime}\end{array}$ & $\begin{array}{l}\operatorname{man} P^{\prime}, \operatorname{man} P^{\prime \prime}, \\
\text { omp }\end{array}$ & manl, mank & - \\
\hline Trehalose & Tre & 3 & 6 & treR $R^{\prime \prime}$ & treT, omp ${ }^{\text {Tre }}$ & treP & - \\
\hline Xylitol & XIt & 2 & 6 & $x \mid t R$ & $x \mid t A B C$ & - & - \\
\hline Ribose & Rbs & 2 & 6 & - & - & - & - \\
\hline Sialic acids & Nan & 1 & 10 & - & nanP, omp $p^{\mathrm{Nan}}$ & - & - \\
\hline Alginate & Alg & 1 & 6 & $\operatorname{alg} R$ & $\operatorname{alg} T$ & - & - \\
\hline Mannitol & $\mathrm{Mtl}$ & 1 & 5 & $m t / R^{\|}$ & $m t / P$ & $\left.m t\right|^{\prime \prime}$ & $m t / X$ \\
\hline Unassigned & - & 19 & 13 & - & - & - & - \\
\hline Total number: & 17 & 19 & 170 & 11 & 34 & 12 & 5 \\
\hline
\end{tabular}

Predicted novel functional assignments verified by targeted experiments are marked by bold type and underlined. The details of all functional assignments summarized in this table are provided in additional files 2 and 3. 


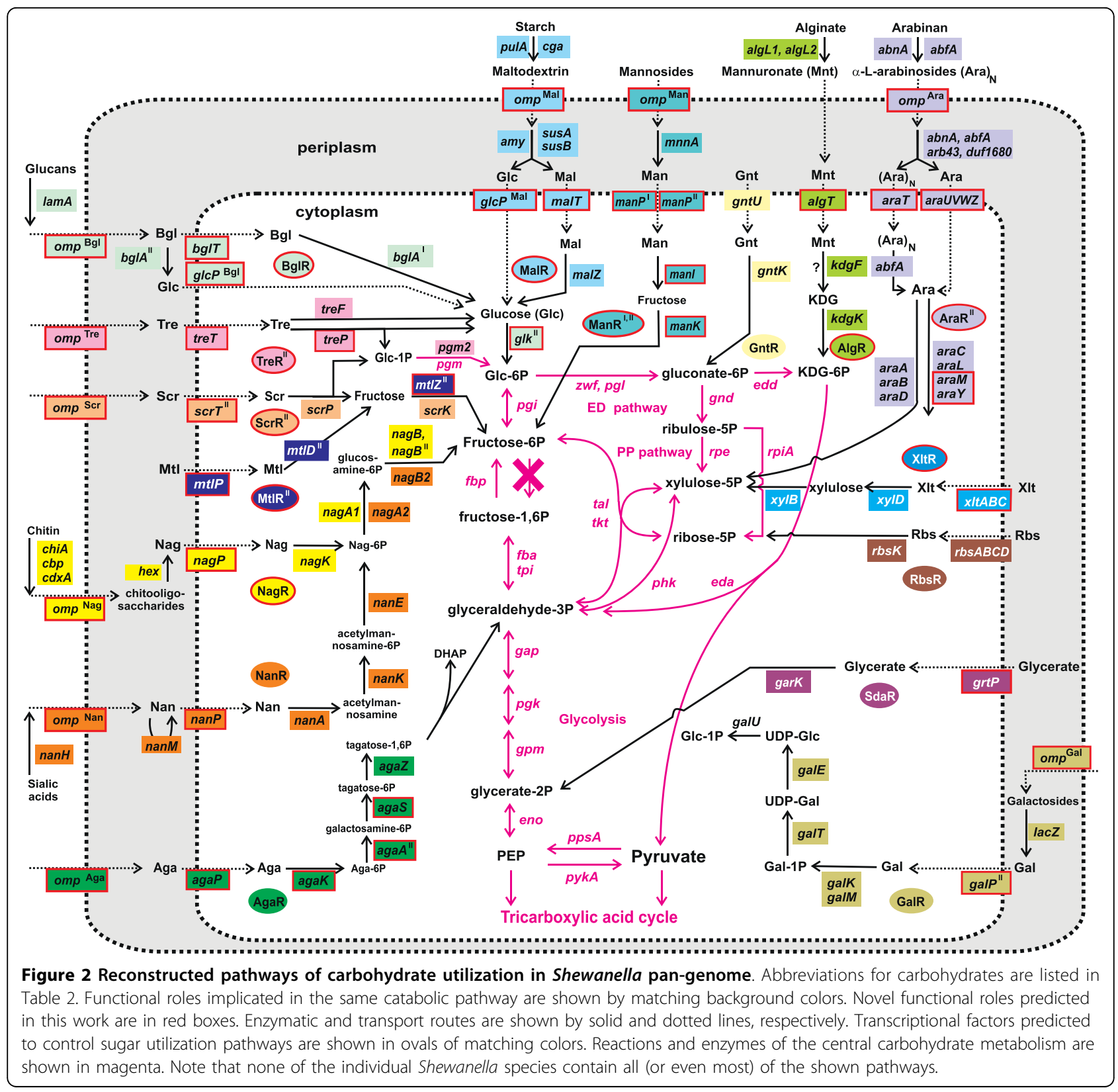

Using the genome context analysis combined with metabolic reconstruction we inferred specific functional assignments for 62 families of isofunctional homologs whose functions were previously unknown or defined only at the level of general class (Table 2, see also additional file 5). The novel functional assignments, including 34 components of transport systems, 11 transcriptional regulators (and corresponding DNA motifs), 12 metabolic enzymes, and 5 auxiliary proteins, are supported by the overall internal consistency of the entire reconstruction where all of the pathways are complete (no gaps or missing genes), and all of the annotated genes are associated with complete pathways (no genes out of context). Selected functional assignments mostly for novel sugar transporters from those pathways that are present in most of the analyzed Shewanella species were tested by targeted biochemical and genetic experiments (for details see next section and additional file 6). An ultimate validation of the entire reconstruction was attained by the experimental testing of growth phenotypes (an ability of a given strain to grow on a specific sugar substrate as a sole source of carbon and energy) predicted by the presence or absence of respective reconstructed pathways. The pathway presence was defined based on the presence of all components of the pathway in a particular genome. This phenotype 
profiling was performed using a "matrix" approach where 14 representative Shewanella species were profiled against a panel of 18 diagnostic sugar substrates (Table 3).

\section{Peripheral sugar utilization pathways}

In this section we describe the key novel aspects of eight reconstructed sugar utilization pathways that were found to be relatively widely distributed in Shewanella genus. The "core" subset includes pathways for utilization of $\mathrm{D}$-glucose, $\mathrm{N}$-acetylglucosamine, $\mathrm{D}$-glycerate, $\beta$-glucosides (present in 14 or more species). The "intermediate" subset includes the sucrose, maltodextrins, D-galactose, and L-arabinose catabolic pathways (conserved in 6 or more species). We also provide the results of experimental validation of selected novel functional roles in five sugar catabolic pathways. The details of the other nine reconstructed pathways for utilization of alginate, gluconate, mannitol, mannosides, $\mathrm{N}$-acetylgalactosamine, ribose, sialic acids, trehalose, and xylitol that were found only in one to four Shewanella strains ("rare" pathways) are described in the additional file 4 . We also report the results of growth phenotype characterization for most of the respective sugar substrates.

\section{$D$-glucose}

(Glc) is utilized by bacteria using either (i) hexose permease and glucokinase, or (ii) PTS ${ }^{\text {Glc }}$ system. The inability to ferment glucose as a carbon source under aerobic conditions was originally attributed to the Shewanella genus, whereas later studies have identified several species that were able to utilize glucose such as $S$. baltica and S. frigidimarina [13-15]. In this study we have tentatively identified respective genes, glucose transporters $g l c P^{\mathrm{Bgl}}$ and $g l c P^{\mathrm{Mal}}$ and glucokinase $g l k^{\mathrm{II}}$, that are conserved in most Shewanella genomes, where they are clustered on the chromosome with the genes from $\beta$-glucoside $(\mathrm{Bgl})$ and maltodextrin $(\mathrm{Mal})$ utilization pathways, respectively (Fig. 3A). These catabolic pathways include several secreted glucosidases (e.g. BglA $^{\mathrm{II}}$, CgA, SusB) generating extracytoplasmic glucose that can be utilized via associated glucose transporters and the glucokinase (see below). The predicted glucose transporters belong to the glucose-galactose permease (GGP) family, and are most similar to the glucose and galactose transporter GluP from Brucella abortus [16] and fucose permease FucP from E. coli. The predicted glucokinase $\mathrm{Glk}^{\mathrm{II}}$ belongs to the ROK family of sugar kinases [17], and is similar to fructokinase Mak, D-allose kinase AlsK, and $\mathrm{N}$-acetylglucosamine kinase NagK from E. coli, and glucokinase GlcK from B. subtilis. Orthologs of both $g l c P^{\mathrm{Mal}} / g l c P^{\mathrm{Bgl}}$ and $g l k^{\mathrm{II}}$ are absent from $E$. coli and other Enterobacteria. The $\mathrm{GlcP}^{\mathrm{Bgl}}$ and $\mathrm{GlcP}^{\mathrm{Mal}}$ transporters in Shewanella were found only in the context of the Bgl and Mal pathways, whereas $\mathrm{Glk}^{\mathrm{II}}$ seems to play a general housekeeping role being conserved in all analyzed Shewanella genomes (see additional data files 2 and 3). In 9 of 19 genomes $g l k^{\mathrm{II}}$ was also found within the Bgl utilization gene cluster, and in 6 of these cases it is a second copy of the gene in the genome.

To confirm the functional assignment of GlcP, we constructed the $\Delta g l c P^{\mathrm{Mal}}$ knockout mutant in Shewanella sp. ANA-3, and tested it for glucose-dependent growth. In contrast to the wild-type ANA-3, the $\Delta g l c P^{M a l}$ strain was unable to grow on D-glucose as a

Table 3 Consistency between the predicted and experimentally determined growth phenotypes of Shewanella spp

\begin{tabular}{|c|c|c|c|c|c|c|c|c|c|c|c|c|}
\hline Strain & Glc & Nag & Grt & $\mathrm{Bgl}$ & $\mathrm{Scr}$ & Mal & Ara & Gal & Gnt & Aga & Tre & Mtl \\
\hline MR1 & $+/ n^{*}$ & $+/ p$ & $+/ p$ & $-/ n$ & $-/ n$ & $+/ n^{*}$ & $-/ n$ & $-/ n$ & $-/ n$ & $-/ n$ & $-/ n$ & $-/ n$ \\
\hline CN32 & $-/ \mathrm{n}$ & $+/ p$ & $+/ p$ & $-/ n$ & $-/ n$ & $-/ n$ & $+/ p$ & $-/ n$ & $-/ n$ & $-/ n$ & $-/ n$ & $-/ n$ \\
\hline W3181 & $-/ n$ & $+/ p$ & $+/ p$ & $-/ n$ & $-/ n$ & $-/ n$ & $+/$ nd & $-/ n$ & -/nd & $-/ n d$ & $-/ n$ & $-/ n d$ \\
\hline OS155 & $+/ p$ & $+/ p$ & $+/ p$ & $+/ p$ & $+/ p$ & $+/ p$ & $-/ n$ & $-/ n$ & $+/ p$ & $-/ n$ & $-/ n$ & $-/ n$ \\
\hline OS185 & $+/ p$ & $+/ p$ & $+/ p$ & $+/ p$ & $+/ p$ & $+/ p$ & $-/ n d$ & $+/ p$ & $+/$ nd & -/nd & $-/ n$ & $-/ n d$ \\
\hline OS195 & $+/ p$ & $+/ p$ & $+/ p$ & $+/ p$ & $+/ p$ & $+/ p$ & $-/ n d$ & $-/ n$ & $+/$ nd & -/nd & $-/ n$ & $-/ n d$ \\
\hline OS223 & $+/ p$ & $+/ p$ & $+/ p$ & $+/ p$ & $+/ p$ & $+/ p$ & $-/ n d$ & $+/ p$ & $+/$ nd & -/nd & $+/ p$ & $-/ n d$ \\
\hline MR7 & $+/ p$ & $+/ p$ & $+/ p$ & $-/ n$ & $+/ p$ & $+/ p$ & $+/ p$ & $-/ n$ & $-/ n$ & $+/ p$ & $-/ n$ & $-/ n$ \\
\hline MR4 & $+/ p$ & $+/ p$ & $+/ p$ & $-/ n$ & $+/ p$ & $+/ p$ & $+/ p$ & $-/ n$ & $-/ n$ & $+/ p$ & $-/ n$ & $-/ n$ \\
\hline ANA3 & $+/ p$ & $+/ p$ & $+/ p$ & $-/ n$ & $+/ p$ & $+/ p$ & $+/ p$ & $-/ n$ & $-/ n$ & $+/ p$ & $-/ n$ & $-/ n$ \\
\hline Sden & $+/ w$ & $+/ p$ & $-/ n$ & $+/ p$ & $-/ n$ & $+/ p$ & $-/ n$ & $-/ n$ & $-/ n$ & $-/ n$ & $-/ n$ & $-/ n$ \\
\hline Sfri & $+/ p$ & $-/ n$ & $+/ p$ & $+/ p$ & $+/ p$ & $+/ p$ & $-/ n$ & $-/ n$ & $-/ n$ & $-/ n$ & $+/ p$ & $+/ n^{*}$ \\
\hline PV4 & $+/ w$ & $+/ p$ & $+/ p$ & $-/ n$ & $-/ n$ & $+/ w$ & $-/ n$ & $+/ \mathrm{w}$ & $-/ n$ & $-/ n$ & $-/ n$ & $-/ n$ \\
\hline Sama & $+/ p$ & $+/ p$ & $-/ n$ & $+/ p$ & $-/ n$ & $+/ p$ & $-/ n$ & $-/ n$ & $-/ n$ & $+/ p$ & $-/ n$ & $-/ n$ \\
\hline
\end{tabular}

Aliases for analyzed Shewanella strains are described in Table 1. Each cell in the table combines the data on the predicted and experimentally determined sugar utilization phenotype. The ability of Shewanella species to grow on a panel of sugar substrates was predicted based on the presence ( + ) or absence (-) of the respective reconstructed pathways in their genomes. The experimental results of growth experiments from this study (see additional data files 7 and 8 ) are: ' $p$ ', positive growth; ' $w$ ', weak growth; ' $n$ ', no growth. Inconsistencies between the predicted and experimentally determined growth phenotypes are in bold and marked by asterisk. 


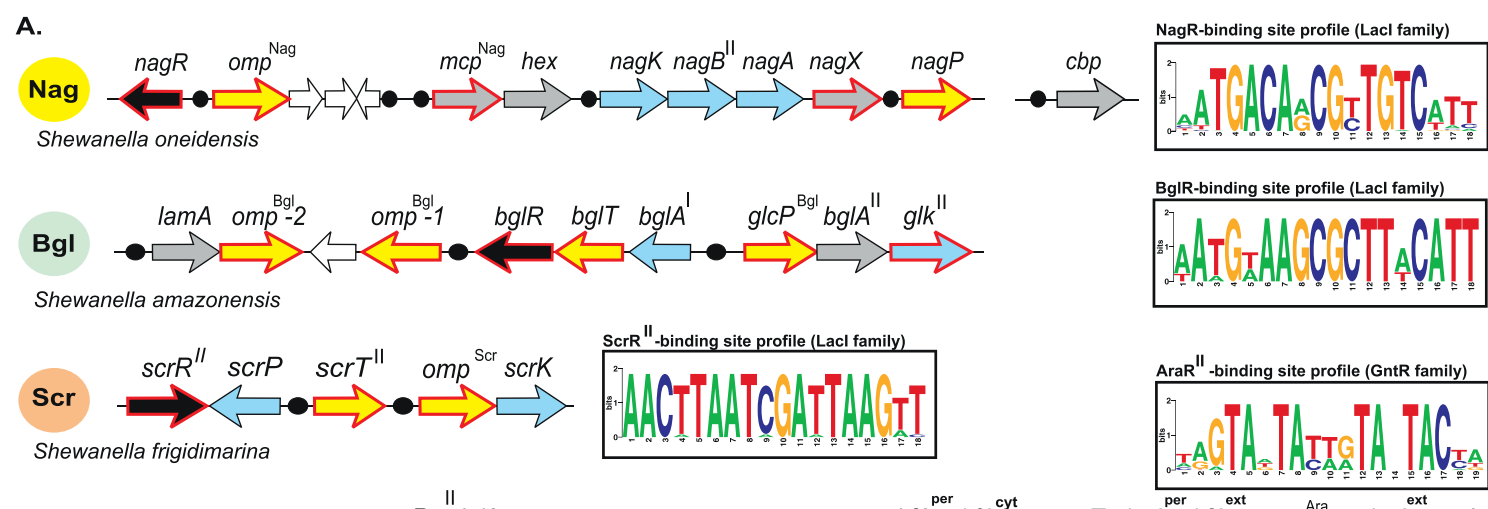

Ara

Shewanella sp. MR-4
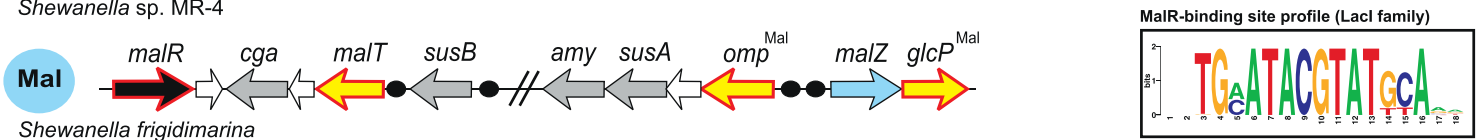

Shewanella frigidimarina
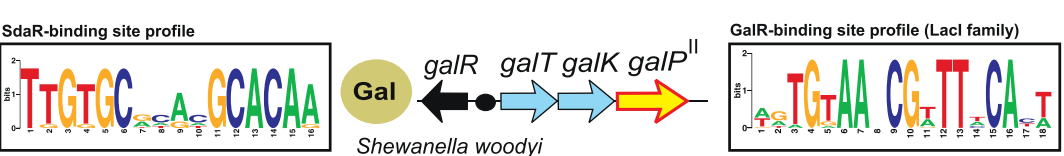

B.
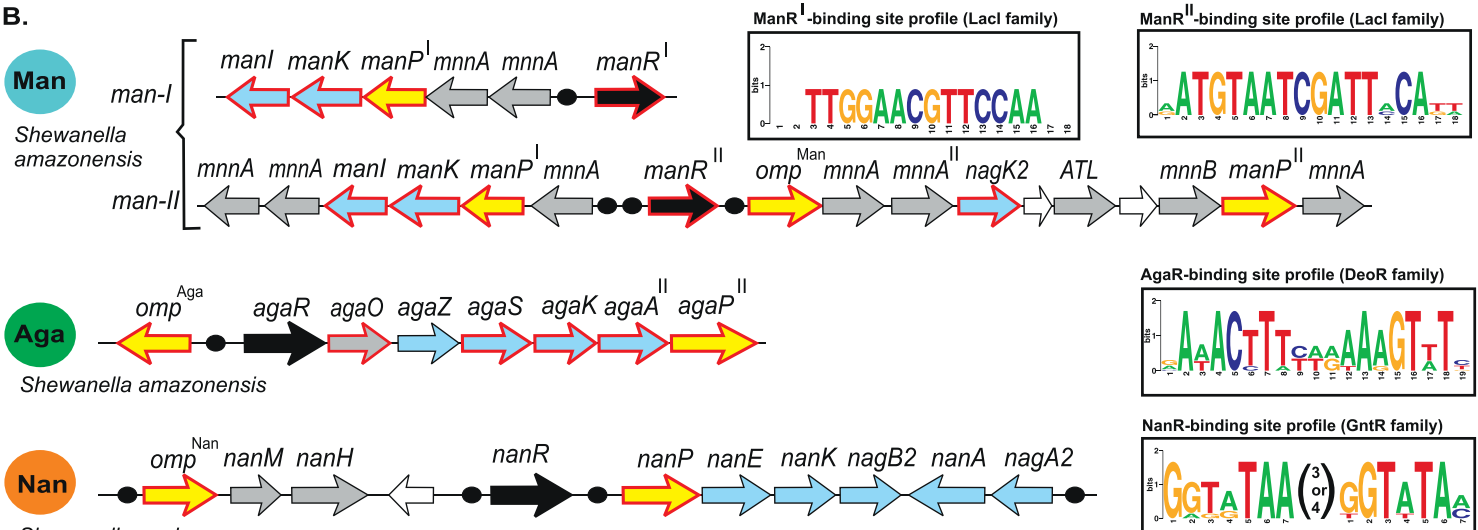

NanR-binding site profile (GntR family)

Shewanella pealeana
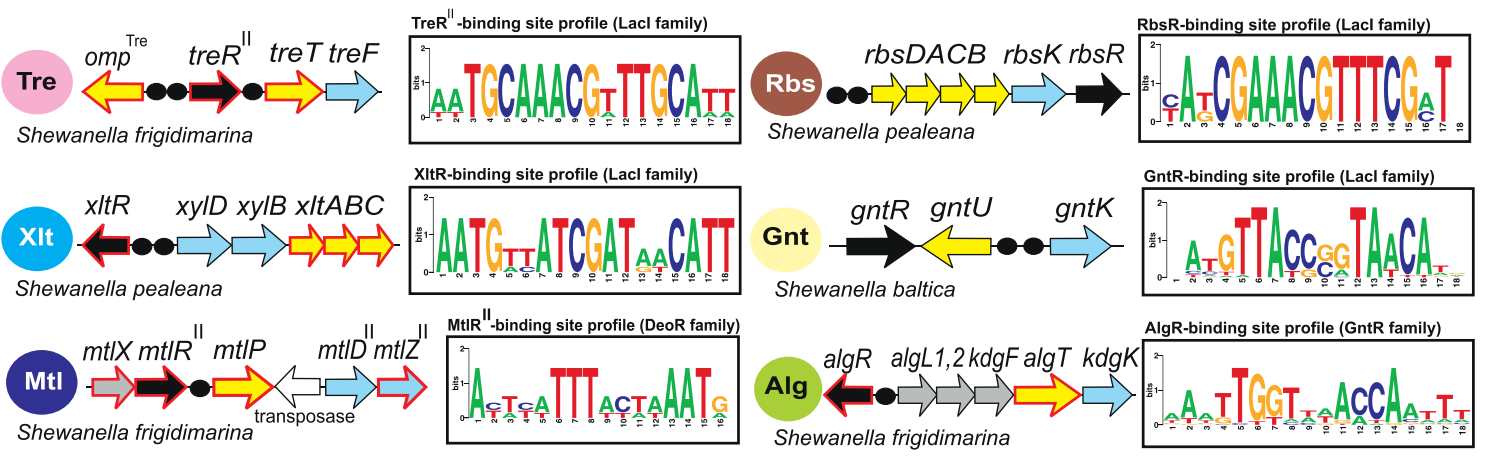

Figure 3 Genomic context and regulons of genes involved in sugar utilization in representative Shewanella genomes. Panel A contains metabolic pathways discussed in the main text as the "core" and intermediate" subsets. Panel B contains "rare' pathways described in the additional file 4. Genes are colored by their functional classification: sugar transport systems, yellow; cytoplasmic enzymes catalyzing biochemical transformations, blue; transcriptional regulators, black; extracytoplasmic sugar hydrolytic enzymes and auxiliary proteins functionally linked to the pathway, gray. Genes with novel functional roles predicted in this work are outlined in red. For each sugar-specific transcription factor, predicted DNA binding sites are shown by black dots and a consensus DNA-binding motif is shown as a sequence logo. 
sole carbon and energy source, confirming the glucose transporter functional assignment (see additional data file 6 for original experimental data). A representative of the predicted glucokinase subfamily $\left(\mathrm{Glk}^{\mathrm{II}}\right)$ from $S$. baltica OS155 was experimentally characterized as a part of our analysis of the Bgl pathway (see below). To assess the functionality of the predicted glucose catabolic pathway we performed phenotypic characterization of Shewanella for growth on D-glucose as a sole carbon and energy source (see additional data files 7 and 8 for the original physiological growth data). Among 14 Shewanella strains tested only $S$. oneidensis and two S. putrefaciens strains were unable to grow on glucose. These results are consistent with the distribution of $g l c P$ transporters in these Shewanella genomes (Table 3, see also additional file 2). The inability of S. oneidensis MR-1 to grow on glucose is most likely due to a frameshift in the $g l c P^{M a l}$ gene[18].

The second (PTS-driven) route of glucose utilization is not expected to support the growth of bacteria (such as Shewanella spp) with the incomplete glycolytic pathway due to the inability of the ED or PP pathways to generate enough phosphoenolpyruvate to compensate for its consumption in the phosphotransferase reaction. This assertion is supported by the observed impaired growth of the E. coli pfk mutant on glucose using PTS ${ }^{\text {Glc }}$ [19]. Therefore, the actual physiological role of Shewanella genes orthologous to the components of the E. coli PTS $^{\text {Glc }}$ system (ptsHI-crr and ptsG) remains a mystery. It would be tempting to speculate that this PTS system is used by Shewanella for glucose consumption in the presence of other substrates contributing to nonglycolytic phosphoenolpyruvate generation. However, our attempts to grow MR-1 on the mixture of lactate and glucose did not confirm this conjecture (data not shown).

\section{$\mathrm{N}$-acetylglucosamine}

(Nag) and chitin catabolic pathway (Fig. 2, see also additional file 9) conserved in most Shewanella spp contains several novel functional roles (Table 2). Previously we have experimentally confirmed the predicted enzymatic activities of novel Nag kinase (NagK) and glucosamine-6-phosphate deaminase $\left(\mathrm{NagB}^{\mathrm{II}}\right)$ and reconstituted the entire three-step biochemical conversion of Nag to fructose-6-phosphate in vitro [9]. We have also confirmed that all of the tested Shewanella strains can grow on Nag as a single source of carbon and energy with the exception of $S$. frigidimarina, which does not contain these genes (Table 3). Here we present additional experimental results supporting functional assignments of the predicted transporter NagP in S. oneidensis MR-1. NagP belongs to the GGP family of transporters and has a limited similarity to the glucose permeases $\mathrm{GlcP}^{\mathrm{Bgl}}$ and $\mathrm{GlcP}^{\mathrm{Mal}}$. We have constructed the S. oneidensis $\Delta n a g P$ targeted deletion mutant and demonstrated the loss of its ability to grow on Nag (see additional data file 6).

The Nag catabolic genes shows their wide distribution in the Alteromonadales lineage, suggesting the presence of the Nag pathway in the common ancestor of this lineage. The absence of Nag pathway in S. frigidimarina is attributed to a loss of the entire chromosomal gene cluster. The observed wide distribution of Nag utilization pathway in Shewanella could be related to their ability to utilize chitin, a highly abundant constituent of aquatic invertebral exoskeleton.

\section{D-glycerate}

(Grt) utilization pathway in Shewanella species involves D-glycerate kinase GarK, transcriptional regulator SdaR, and a novel D-glycerate permease termed here GrtP (Fig. 2, see also additional file 9). In E. coli, GarK is involved in the glucarate/galactarate catabolic pathway, generating 2-phosphoglycerate as product, and SdaR is a common transcriptional regulator of this pathway [20]. Most of the glucarate/galactarate utilization genes are absent in Shewanella species. The only exception is a garK gene ortholog, which was found in 17 Shewanella strains in a conserved operon with a hypothetical gene encoding the predicted D-glycerate transporter GrtP (see additional data files 2 and 3). The grtP-garK operon is clustered on the chromosome with an $s d a R$ gene ortholog. A comparative genomic reconstruction of the SdaR regulon in $\gamma$-proteobacteria allowed us to predict a candidate SdaR-binding site located upstream of the grtP-garK operon in Shewanella genomes (Fig. 3A).

The predicted D-glycerate uptake transporter GrtP belongs to the gluconate permease family and has orthologs in Pseudomonadales and Vibrionales but not in E. coli or other Enterobacteria. The predicted function of GrtP was confirmed by the loss of ability of the S. oneidensis $\triangle$ grtP strain to grow on D-glycerate as a sole carbon and energy source (see additional data file 6). Of the 14 Shewanella strains, all but 2 (S. denitrificans and $S$. amazonensis) could grow on D-glycerate (see additional data files 7 and 8 ), which is fully consistent with the distribution of the grtP-garK operon among the analyzed Shewanella genomes (Table 3). The natural source of $\mathrm{D}$-glycerate in aquatic environments is yet to be elucidated.

\section{$\beta$-glucoside}

'Bgl' utilization pathway revealed in 9 Shewanella species involves $\beta$-glucanase LamA, two $\beta$-glucosidases BglA ${ }^{\mathrm{I}}$ and $\mathrm{BglA}^{\mathrm{II}}$, two novel $\beta$-glucoside transporters BglT and $\mathrm{Omp}^{\mathrm{Bgl}}$, a novel LacI-type transcriptional regulator BglR, glucose permease $\mathrm{GlcP}^{\mathrm{Bgl}}$ and ROK-type glucokinase Glk ${ }^{\mathrm{II}}$ (Fig. 2, see additional data files 2, 3 and 9). The previously described $\mathrm{Bgl}$ catabolic pathways use PTS-type transport systems and 6-phospho- $\beta$-glucosidases (as in 
B. subtilis and E. coli) or ABC-type transport systems and $\beta$-glucosidases (as in Streptomyces spp and Archaea) [21]. The predicted $\beta$-glucoside uptake transporter BglT is a member of the glycoside-pentoside-hexuronide (GPH) transporter family, and has orthologs in other Alteromonadales species. Homologs of BglT in Enterobacteria (e.g. YicJ and YagG from E. coli that show 40-44\% sequence similarity to BglT) are predicted xyloside transporters regulated by the xylose activator XylR [22]. Omp ${ }^{\text {Bgl }}$ belongs to the TonB-dependent outer membrane transporter (TBDT) family, which includes proteins involved in high-affinity binding and energy-dependent uptake of various substrates (including oligosaccharides) into the periplasm [23]. The predicted Bgl-specific transporter $\mathrm{Omp}^{\mathrm{Bgl}}$ is a nonorthologous replacement of the Bgl-specific outer membrane porin BglH from E. coli. Comparative genomic reconstruction of the BglR regulon allowed us to predict candidate BglR-binding sites located upstream of the divergently transcribed $b g l A^{I}-b g l T-b g l R$ and $g l c P^{B g l}-b g l A^{I I}$ operons, and upstream of the $o m p^{B g l}$ gene (Fig. 3A). Thus the predicted novel transporters are positionally clustered and co-regulated with other Bgl catabolic genes.

The reconstructed Bgl pathway in Shewanella involves three glucosidases, two of which, LamA and BglA ${ }^{\mathrm{II}}$, are predicted to be secreted outside of the cell and to the periplasm, respectively, whereas $\mathrm{BglA}^{\mathrm{I}}$ is likely a cytoplasmic enzyme. We propose that $\beta$-glucoside-containing glucans are first degraded by extracellular endo$\beta$-1,3-glucanase Lam $A$, the resulting oligo- $\beta$-glucosides are transported to the periplasm by $\mathrm{Omp}{ }^{\mathrm{Bgl}}$, and subsequently utilized by BglA ${ }^{\text {II }}$ to produce D-glucose and shorter $\beta$-glucosides (e.g., cellobiose, gentibiose). The latter products are taken up by the predicted BglT transporter into the cytoplasm where they are finally hydrolyzed by the $\mathrm{BglA}^{\mathrm{I}}$ enzyme. D-glucose is taken up by the predicted glucose transporter $\mathrm{GlcP}^{\mathrm{Bgl}}$ and phosphorylated by the Glk ${ }^{\mathrm{II}}$ glucokinase (Fig. 2).

The newly predicted $\beta$-glucoside BglT transporter was experimentally confirmed by genetic complementation in E. coli $\triangle b g l F$ mutant (a knockout of cellobiose PTS system). This strain, when transformed by an expression vector containing an operon $b g l A^{I}$ - $b g l T$ cloned from $S$. baltica OS155 gained the ability to grow on minimal medium with cellobiose as the sole carbon and energy source. In the same conditions, no growth was observed for this strain transformed by vector only or by the plasmids containing single genes, $b g l A^{I}$ or $b g l T$ (see additional data file 6).

The predicted glucokinase activity of the S. baltica OS155 $g l k^{\mathrm{II}}$ gene product was confirmed by an in vitro enzyme assay. Recombinant purified Glk ${ }^{\mathrm{II}}$ exhibited a broad substrate specificity at $37^{\circ} \mathrm{C}$ with the highest activity with D-glucose $(22 \mu \mathrm{mol} / \mathrm{mg} / \mathrm{min})$, and an appreciable activity with some other tested hexoses including D-mannose, D-fructose, D-glucosamine, and D-mannosamine (see additional data file 6).

The results of the phenotype profiling of 14 Shewanella strains showed that only half of them (four S. baltica species, S. amazonensis, S. frigidimarina, and $S$. denitrificans) were able to grow on cellobiose as a sole carbon and energy source (see additional data files 7 and 3). This result is consistent with the distribution of the respective genes in the analyzed Shewanella genomes (Table 3).

The Bgl catabolic genes are sparsely distributed among Shewanella species (Fig. 4) and many other species from the Alteromonadales lineage. Therefore, it might be an ancestral pathway predating the speciation of Alteromonadales that could have been independently lost several times within the Shewanella genus. The species-specific loss of Bgl pathway by many Shewanella could be linked to a particular ecophysiology of their habitat. For example, S. pealeana which colonizes the accessory nidamental gland of the squid may not have access to Bgl substrate and thus lost the respective gene cluster from its chromosome [15].

\section{Sucrose}

(Scr) utilization pathway in 8 Shewanella species involves sucrose phosphorylase ScrP, fructokinase ScrK, two novel sucrose transporters ( $\mathrm{ScrT}^{\mathrm{II}}$ and $\mathrm{Omp}{ }^{\mathrm{Scr}}$ ), and a novel LacI-type transcriptional regulator $\mathrm{ScrR}^{\mathrm{II}}$ (Fig. 2; see also see additional data files 2, 3 and 9). This pathway variant is quite different from the canonical pathway known in Enterobacteria, which includes the sucrose-specific PTS-driven uptake and phosphorylation followed by sucrose-6-phosphate hydrolase [24]. An alternative variant of the Scr pathway previously described in Bifidobacterium spp includes sucrose permease ScrT from the MelB melibiose transporter family and sucrose phosphorylase [25]. The major disctinction in Shewanella is a nonorthologous replacement of ScrT by a predicted transporter $\mathrm{ScrT}^{\mathrm{II}}$ of the GGP family of sugar transporters. The novel sucrose regulator $\operatorname{ScrR}^{\mathrm{II}}$ in Shewanella is another nonorthologous replacement of the previously characterized ScrR repressor from other bacteria. A genomic reconstruction of the $\mathrm{ScrR}^{\mathrm{II}}$ regulon allowed us to predict its candidate binding sites upstream of the divergently transcribed $s c r P$ and $s c r T^{I I}$ genes, as well as upstream of the $o m p^{S c r}$-scrK operon (Fig. 3A). The predicted Scr-specific TBDT Omp ${ }^{\mathrm{Scr}}$ is functionally equivalent to the Scr-specific outer membrane porin ScrY from Enterobacteria, and is presumably involved in the uptake of sucrose into the periplasm[26].

The ScrT ${ }^{\mathrm{II}}$ transporter was validated by complementation in E. coli $\mathrm{K}-12$, which lacks the Scr pathway and is unable to utilize sucrose (see additional data file 6). Two 


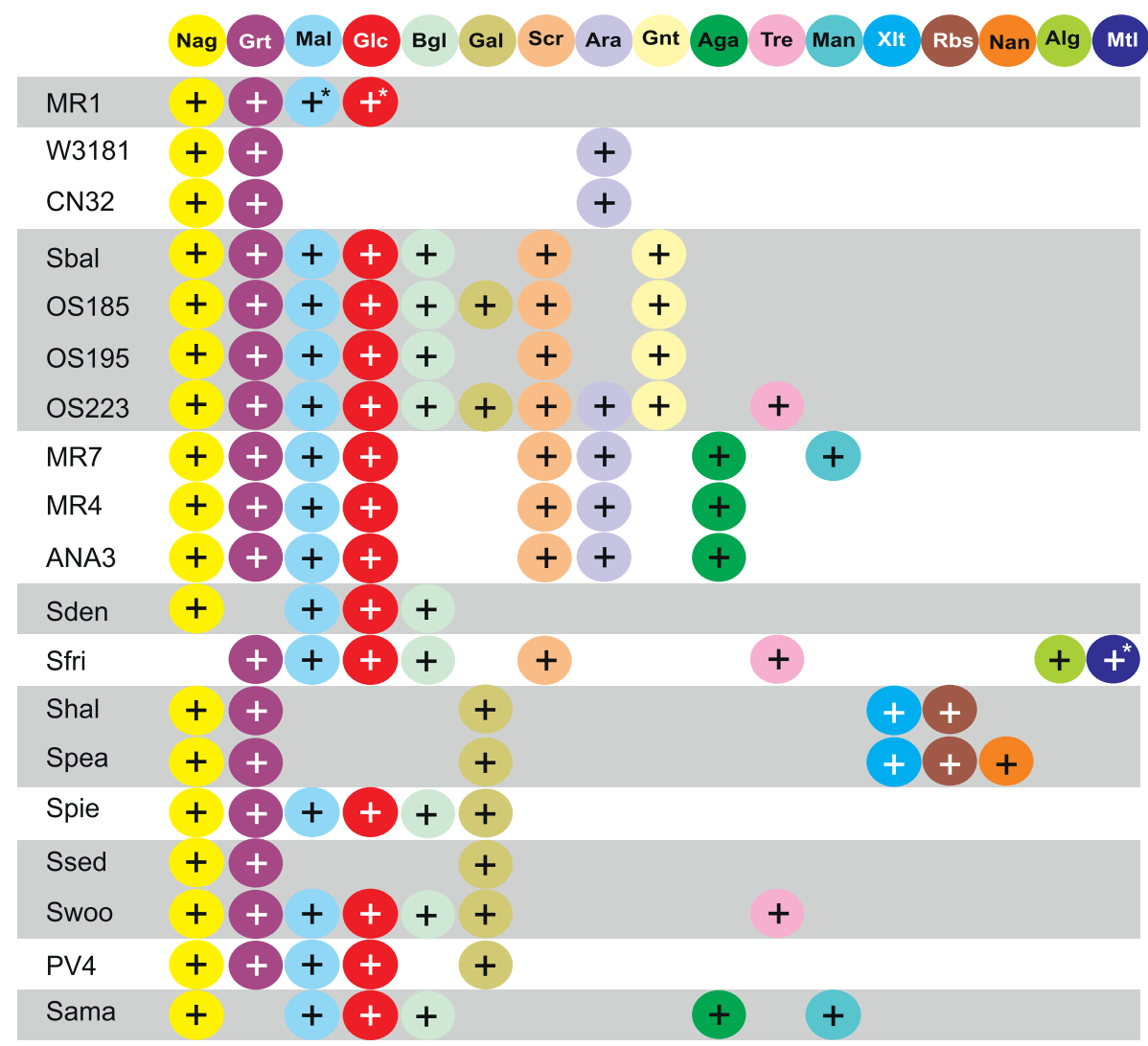

Figure 4 Distribution of 17 sugar utilization pathways encoded in 19 Shewanella genomes. Shewanella species abbreviations are as described in Table 1. Asterisks indicate cases when a pathway is impaired by the presence of an insertion sequence element or a frameshift mutation and, thus, deemed nonfunctional.

divergently transcribed genes from $S$. frigidimarina, $s c r T^{I I}$ and $\operatorname{scr} P$, were expressed in E. coli under the control of an endogenous promoter in their common intergenic region. As a negative control, we used an empty vector as well as single $s c r T^{I I}$ or scrP genes expressed in the same strain. The cell growth was monitored in a minimal medium with sucrose as the only carbon and energy source. We have found that only the cells carrying both $s c r T^{I I}$ and scrP were able to grow on sucrose providing an experimental verification of the reconstructed Scr utilization pathway.

The growth phenotype profiling of 14 Shewanella species demonstrated that eight of them (Shewanella sp. ANA-3, MR-4, MR-7, four S. baltica strains, S. frigidimarina) are able to grow on sucrose (see additional data files 7 and 8). These results are in agreement with the presence of the respective genes in these 8 Shewanella genomes (Table 3).

The Scr catabolic genes are present in a large group of Shewanella species (Fig. 4) and in some other species from the Alteromonadales lineage. The most parsimonious scenario suggests that the Scr pathway was present in a common ancestor of the Shewanella genus and that it has been independently lost several times in the evolutionary history of Shewanella. Distribution of the Scr pathway among the Shewanella species provides another possible link to their ecophysiology, the availability of plant-derived sucrose in their respective ecologic niches.

\section{Maltodextrin}

(Mal) utilization gene locus identified in 14 Shewanella species contains the amy, cga, susA, susB, and malZ genes encoding orthologs of previously characterized starch and maltodextrin hydrolytic enzymes, as well as genes encoding novel transporters termed MalT, $\mathrm{GlcP}^{\mathrm{Mal}}$ and $\mathrm{Omp}^{\mathrm{Mal}}$, and a novel LacI-type transcriptional regulator MalR (see additional data file 2). The reconstructed Mal catabolic pathway in Shewanella (Fig. 2) uses a predicted novel sodium:solute symporter MalT (a homolog of the melibiose permease MelB of E. coli) instead of the maltose ABC transporter MalEFGK previously described in E. coli [27]. The outer membrane maltose/maltodextrin uptake in Shewanella is presumably mediated by a novel TBDT Omp ${ }^{\text {Mal }}$, the functional equivalent of $E$. coli maltoporin LamB[26]. The novel glucose transporter $\mathrm{GlcP}^{\mathrm{Mal}}$ is a close paralog $(67 \%$ 
identity) of the predicted glucose transporter $\mathrm{Glc}^{\mathrm{Bgl}}$ from the Bgl utilization pathway in Shewanella. We propose that $\mathrm{D}$-glucose is produced in the periplasm by the action of maltodextrin $\alpha$-glucosidases and transported into the cytoplasm by $\mathrm{GlcP}^{\mathrm{Mal}}$. A comparative genomic reconstruction of the MalR regulon allowed us to predict candidate MalR-binding sites located upstream of the divergently transcribed $o m p^{\mathrm{Mal}}$ and $m a l Z-g l c P^{\mathrm{Mal}}$ operons, as well as upstream of the $\operatorname{susB}$ and malT-cga operons (Fig. 3A).

The growth phenotype characterization of 14 Shewanella species demonstrated that 11 of them (all except MR-1 and two S. putrefaciens strains) are able to grow on maltodextrin as a sole carbon and energy source (see additional data files 7 and 8 ). These phenotypic results are in agreement with the genomic reconstruction of the Mal pathway (Table 3). The inability of S. oneidensis MR-1 to grow on maltodextrin is attributed to significant genetic perturbations within the Mal utilization gene loci (e.g. $g l c P^{\mathrm{Mal}}$ and sus $A$ are pseudogenes with frameshifts, whereas $o m p^{\text {Mal }}$ is interrupted by an insertion element [18]).

The Mal catabolic genes are widely distributed among Shewanella species (Fig. 4) and several other species from the Alteromonadales lineage. Such phyletic pattern suggests the presence of an ancestral pathway in the common ancestor of the Shewanella genus and independent species-specific pathway losses. Interestingly, while the Mal pathway in S. oneidensis MR-1 demonstrates genetic signs of decay (see above), two closely related $S$. putrefaciens strains have completely lost the Mal pathway genes. The loss of the Mal catabolic genes in some Shewanella spp. is most likely caused by a significant shift in available nutrient composition in their habitat, e.g. the absence of plant materials as a source of maltodexrins.

\section{L-arabinose}

(Ara) and arabinoside utilization gene locus identified in six Shewanella species contains more than 20 genes. Nearly half of these genes are similar to previously characterized Ara catabolic genes whereas others are novel (see additional data files 2 and 9). The reconstructed Ara catabolic pathway in Shewanella involves the following predicted functional roles: arabinose $A B C$ transporter AraUVWZ, two arabinoside transporters AraT and Omp ${ }^{\text {Ara }}$, a novel GntR-type transcriptional regulator $\mathrm{AraR}^{\mathrm{II}}$, L-arabinose mutarotase AraM, and Ara 1-dehydrogenase AraY (Table 2, Fig. 2).

Two alternative routes of Ara utilization are known in bacteria. The major one present in E. coli and B. subtilis depends on $\mathrm{L}$-arabinose isomerase AraA, L-ribulokinase $A r a B$, and L-ribulose-phosphate epimerase AraD, whereas the alternative Ara pathway characterized in Azospirillum brasiliense proceeds through $\mathrm{L}$-arabinose dehydrogenase, arabinolactonase, and L-arabonate dehydratase [28]. In addition to the araBAD genes encoding the conventional Ara utilization pathway through Lribulose, the Ara metabolic locus in Shewanella contains two genes from the alternative Ara pathway, namely Larabonate dehydratase $\operatorname{araC}$ and arabinolactonase araL, although other genes from the alternative pathway are missing (see additional data file 9). Based on genome context and distant homology analysis we have predicted the gene $\operatorname{ara} Y$, which is similar to D-xylose 1dehydrogenase from Caulobacter crescentus (45\% similarity), to be the missing L-arabinose 1-dehydrogenase. A member of aldose 1-epimerase family encoded in the Ara gene cluster was tentatively assigned the functional role Ara mutarotase (AraM), which interconverts alpha and beta anomers of L-arabinose.

The predicted $A B C$-type arabinose uptake transporter system AraUVWZ is similar to the hypothetical sugar transporter YtfQRST from E. coli (58\% similarity) and to the ribose transport systems in E. coli and B. subtilis. The predicted TBDT Omp ${ }^{\text {Ara }}$ and the GPH-family transporter AraT are presumably involved in the uptake of arabinosides through the outer and inner membrane, respectively. Comparative genomic reconstruction of the $\mathrm{AraR}^{\mathrm{II}}$ regulon allowed us to predict its candidate binding sites located in the likely regulatory regions of most operons in the ara locus (Fig. 3A). Thus, the predicted novel transporters are both positionally clustered and co-regulated with the other Ara catabolic genes (see additional data files 2 and 3 ).

The functionality of the predicted arabinose utilization pathway is supported by the growth phenotype profile of Shewanella spp on L-arabinose (see additional data files 7 and 8), which correlates perfectly with the presence/absence of the Ara catabolic genes (Table 3).

The Ara catabolic genes are present in a large group of Shewanella species (Fig. 4), among which MR-1 and three S. baltica strains have lost the complete ara gene cluster. Outside of the Shewanella genus the most similar ara catabolic gene clusters were found in two other $\gamma$-proteobacteria, the polysaccharide-degrading marine bacterium Saccharophagus degradans and the plant cell wall-degrading soil bacterium Cellvibrio japonicus. Arabinose is an important component of plant cell wall. Therefore, the distribution of the Ara catabolic pathway among Shewanella species may also reflect differences in the availability of plant-derived arabinose in their environments.

\section{D-galactose}

(Gal) metabolism genes encoding galactokinase GalK, mutarotase GalM, UDP-glucose epimerase GalE, and UTP-glucose-1-phosphate uridylyltransferase GalU are conserved in all Shewanella genomes (see additional data file 2). Utilization of extracellular galactose or 
galactosides requires specific transport systems and sugar hydrolases. The galKM locus in S. halifaxensis, $S$. loihica, S. pealeana, S. piezotolerans, and S. sediminis involves two additional genes, galP $P^{I I}$ and $l a c Z$, that are presumably involved in galactose and lactose catabolism. The predicted galactose permease GalP ${ }^{\mathrm{II}}$ belongs to the SSF superfamily and is similar to the myo-inositol and glucose transporters from mammals (34\% identity). The $\beta$-galactosidase LacZ has a candidate signal peptide cleavage site and is presumably a secreted enzyme. We propose that $\beta$-galactosides are degraded in the periplasm by LacZ, and the resulting $D$-galactose residues are taken up by GalP ${ }^{\mathrm{II}}$ transporter (Fig. 2). The omp ${ }^{\text {Gal }}$ lacZ-galTK-galP ${ }^{\mathrm{II}}$-galE operon in two $S$. baltica strains (OS185 and OS223) encodes an additional novel transporter from TBDT family, named Omp ${ }^{\text {Gal }}$, which is likely involved in lactose uptake through the outer membrane.

The galTKP $P^{I I}$ operon identified in S. woodyi is presumably involved in D-galactose monosaccharide utilization, because of the absence of candidate galactoside hydrolase genes in this genome. An ortholog of the transcriptional regulator gene galR from E. coli is clustered with the galTKP $P^{I I}$ operon in $S$. woodyi and with the omp ${ }^{\mathrm{Gal}}$-lacZ-galTK-galP ${ }^{\mathrm{II}}$-galE operon in two $S$. baltica strains, and candidate GalR-binding sites were identified in their corresponding upstream regions (Fig. 3).

The growth phenotype characterization of 14 Shewanella species demonstrated that only three strains (S. loihica PV-4, and two S.batica strains) are able to grow on D-galactose (see additional data files 7 and 8). This pattern is consistent with the distribution of the predicted galactose permease gene galP $P^{I I}$ in the analyzed Shewanella genomes (Table 3).

A mosaic distribution of the galP ${ }^{I I}$ genes in two groups of the Shewanella spp., and the presence of their orthologs in several other marine bacteria from the Alteromonadales lineage suggest multiple gene loss events in the evolutionary history of the Gal catabolic pathway in the Shewanella genus. The Gal utilization genes could have been lost in those Shewanella species that do not share the same ecological niche with some of the marine animals that are thought to provide a natural source of galactose and $\beta$ galactosides.

\section{Novel sugar utilization pathway variants in Shewanella}

The reconstructed peripheral pathways in Shewanella spp contain 62 variations distinguishing them from those previously described in model species, thus providing a vivid illustration of the aforementioned intrinsic variability of the sugar utilization machinery (see additional data file 5). Most common are numerous cases of nonorthologous gene replacements (corresponding to novel FIGfams), when a functional role is encoded by a gene that is not orthologous (and, in many cases, not homologous) to any of the previously known genes of the same function [29]. In our analysis, such deviations as well as a few cases of alternative biochemical routes were initially recognized as inconsistencies or gaps (missing genes) in reconstructed pathways. Such gaps were filled-in by the most likely gene candidates revealed by genome context analysis (e.g. those functionally coupled with canonical genes of the respective pathways via operons and/or regulons). This analysis also allowed us to extend some of the pathways by adding components that would not be commonly perceived as genuine gaps, such as transcriptional regulators and transporters.

Althogh this study is restricted to Shewanella spp., most of the newly assigned protein families contain multiple representatives outside the Shewanella genus, thus contributing to the reconstruction of respective pathways in a variety of species. It is worth noting that the addition of 62 novel FIGfams led to an appreciable (> 12\%) expansion of our original collection of 480 FIGfams associated with sugar utilization subsystems (Fig. 1). Similar analysis applied to new groups of species may reveal additional FIGfams and their combinations involved in the carbohydrate utilization machinery, thus iteratively expanding the entire collection. Of no less importance is the fact that nearly all of the newly assigned genes were originally identified as distant homologs of previously characterized genes with distinct but related functions. For example, a novel Aga kinase was identified as a distant homolog of other known sugar kinases; the novel D-glycerate transporter - as a homolog of E. coli D-gluconate permease; the novel BglR repressor - as a homolog of many sugar-specific regulators from the LacI family. Although this observation obviously reflects intrinsic limitations of homology-based predictions, it also delivers a more important message that our knowledge of protein families involved in the carbohydrate utilization is close to saturation at the level of general class functions recognized by homology-based methods. Otherwise, at least some of the gap-filling gene candidates predicted by genome context analysis would have shared no homology with previously known components from the collection. Not a single example of that kind was observed in this study. Moreover, not a single gap has remained in any of the 17 reconstructed sugar utilization pathways clearly supporting the above interpretation.

Characteristic features of the Shewanella sugar catabolome contrasted with E. coli and other Enterobacteria are described below. 


\section{Carbohydrate uptake}

strategies appear to be strikingly different between Shewanella and Enterobacteria. Overall, 34 gene families encoding novel versions of sugar uptake systems were identified in Shewanella spp. Most notably, in contrast to an extensive repertoire of 21 PTS systems actively used by E. coli for the uptake of various sugars [30], most Shewanella species contain only one PTS system of unclear physiological role. As already discussed, the lack of Pfk genes blocks the conventional glycolytic route in Shewanella making the ED pathway the only feasible way of hexose utilization. The inability of the latter pathway to sustain energy requirements of PTS systems (due to phosphoenolpyruvate regeneration balance) provides a rationale for their absence in Shewanella spp. Thus, the uptake of Glc, Man, Scr, Tre, Nag, and Aga in E. coli is mediated by dedicated PTS systems, whereas in Shewanella species these sugars are transported by predicted novel permeases of the GGP family, GlcP, ManP, ScrT ${ }^{\mathrm{II}}$, TreT, NagP, AgaP ${ }^{\mathrm{II}}$, respectively. A combination of inner membrane transporters from the GGP and several other sugar permease families with committed outer membrane transporters of the TBDT family appears to be the predominant strategy of sugar uptake in Shewanella (Fig. 2). Committed TBDT outer membrane transporters were mapped for 10 of the 17 reconstructed sugar utilization pathways in Shewanella species based on their occurrence within respective operons and regulons (Table 2). The predicted Shewanella TBDT transporters are functionally equivalent to sugar-specific outer membrane porins of Enterobacteria (e.g. BglH, ScrY, LamB, NanC). However, in contrast to porins mediating transport across the outer membrane by passive diffusion, TBDT transporters employ a unique energizing mechanism utilizing the TonB complex and the proton-motive force of the cytoplasmic membrane. It is tempting to speculate that a relative abundance of TBDT transporters in Shewanella and some other environmental Proteobacteria reflects their rather limited access to carbohydrates as compared to Enterobacteria.

\section{Transcriptional regulation}

is another highly variable aspect of the sugar utilization machinery. Indeed, 11 of the 17 transcriptional regulators tentatively associated with Shewanella sugar utilization pathways are nonorthologous to their counterparts previously characterized in E. coli and other species (Table 2). Moreover, 9 of these transcription factors were recruited from structurally unrelated protein families, and they are predicted to bind to completely distinct DNA motifs. For example, the transcriptional repressor NagR of the Nag utilization pathway in Shewanella belongs to the LacI family, whereas a functionally equivalent $\mathrm{NagC}$ regulator of $E$. coli belongs to the
ROK family. Therefore, a functional repertoire of coregulated genes appears to be generally better conserved between species than the respective transcription factors and their DNA binding motifs [31]. Members of the LacI family are most abundant among the regulators of sugar catabolism in Shewanella. They control 10 of the 17 reconstructed pathways, whereas the remaining pathways are regulated by the members of GntR and DeoR families (Fig. 3). The majority of genes from sugar catabolic pathways were identified as candidate members of respective sugar catabolic regulons in Shewanella genomes (see additional data file 3).

\section{Sugar catabolic enzymes}

are far less variable between species than associated transporters or regulators. In case of Shewanella, the most notable variations are associated with sugar phosphorylation, which is at least partially due to a functional replacement of the uptake-coupled phosphorylation (characterstic of PTS) by a combination of permease and kinase or, less frequently, phopshorylase (for disaccharides). Thus, in the aforementioned $\mathrm{Nag}$ and Aga pathways, novel sugar kinases NagK and AgaK combined with respective permeases (NagP and AgaP) are employed in Shewanella spp rather than canonical PTS systems. In a novel variant of Scr utilization pathway predicted and experimentally validated in S. frigidimarina (see additional data file 6), the hydrolysis of sucrose upon ScrT-mediated uptake and phosphorylation of its glucose moiety is performed by the sucrose phosphorylase (ScrP). This single-enzyme transformation is a functional replacement of two consecutive enzymatic reactions, phosphotransferase by PTS (ScrA) followed by sucrose-6-phosphate hydrolase (ScrB), in a canonical version of the pathway described in Enterobacteria [24]. Nonorthologous replacements appear to be quite common for sugar kinases that are known to occur in a number of distinct protein families. For example, the glucokinase $\mathrm{Glk}^{\mathrm{II}}$ of Shewanella belongs to the ROK family, whereas its functional counterpart in E. coli and other Enterobacteria belongs to the bacterial glucokinase family.

\section{Discussion}

Most heterotrophic bacteria are capable of utilizing at least some carbohydrates as a source of carbon and energy via a matching repertoire of sugar catabolic pathways. Such pathways that include specialized transporters and intracellular enzymes catalyzing biochemical transformations of a particular sugar into one of the common CCM intermediates often form operons controlled by committed transcription factors. Despite our advanced understanding of sugar catabolic pathways in a few model bacteria, their genomics-based projection across a variety of species from other taxonomic groups 
is quite challenging due to the intrinsic variability of the carbohydrate utilization machinery. To address this challenge we have established a subsystems-based comparative genomic approach (Fig. 1), and assessed its utility by building a genomic encyclopedia of carbohydrate catabolism (sugar catabolome) in the Shewanella genus. This analysis covered 19 complete genomes of diverse Shewanella species isolated from various aquatic habitats. The key components of our approach include: (i) using a collection of protein families capturing the current knowledge of the microbial carbohydrate utilization machinery for homology scanning of target genomes and preliminary identification of gene candidates; (ii) analyzing genomic and functional contexts to recognize functional equivalents of previously characterized genes and to predict novel genes and pathway variants; (iii) validating selected bioinformatic predictions by a combination of biochemical, genetic and physiological experiments.

One of the critical factors that contributed to the successful outcome of this analysis was the availability of a large set of completely sequenced genomes (largely due to the DOE Joint Genome Institute, http://www.jgi.doe. gov/) representing a substantial phylogenetic, geographic and ecophysiological diversity within an otherwise compact taxonomic group - Shewanella genus [1]. This was particularly instrumental for mapping and prediction of regulons as well as for reliably establishing orthology relationships within protein families containing multiple paralogs. In addition to improving our knowledge of genomics, functional organization and evolution of the sugar catabolome, this study confirmed the efficiency of the established approach, which is scalable and applicable to other groups of microorganisms. A systematic application of this approach to a growing number of divergent bacterial lineages would allow us to rapidly expand the current genomic knowledgebase and establish the capability of highly accurate automated annotation and assertion of sugar catabolic pathways in all bacteria with completely sequenced genomes. Moreover, a fine-grain functional annotation of all protein families (including those with multiple branches and paralogs) that comprise carbohydrate utilization machinery in complete genomes will enable accurate recognition of the corresponding functions and pathway variants in metagenomic samples.

An overall internal consistency of the reconstructed catabolic machinery confirmed the efficiency of the bioinformatic workflow used in this study. The current version of the genomic encyclopedia of carbohydrate utilization pathways of the Shewanella genus has neither obvious gaps such as "missing genes" (required by functional context but not identified in the genomes), nor inconsistencies such as "disconnected genes" (those that were functionally assigned but lack any functional context). The only notable exception is an already mentioned putative PTS system whose function remains obscure despite its high similarity with $E$. coli glucose PTS. Additional support of the developed genomic reconstruction was provided by direct experimental testing of selected bioinformatic predictions using both in vitro assays of purified recombinant enzymes and in vivo phenotype assessment of gene knockouts in Shewanella and genetic complementation in E. coli. Despite the overall successful outcome and utility of these experiments, the scale of this reconstruction makes a traditional targeted verification of individual conjectures rather impractical. On the other hand, a predicted pattern of presence/absence of certain sugar utilization pathways within a representative group of species may be seamlessly translated to a predicted testable phenotype, the ability/inability of these species to metabolize respective sugars.

We assessed the panel of 14 Shewanella species for the aerobic growth on a series of 18 sugars used as a single source of carbon and energy (see additional data files 7 and 8). The results of the extended growth profiling in this study revealed a remarkable consistency between predicted and observed phenotypes for the entire matrix of tested strains and sugars despite some strain-to-strain variations in the growth rate and efficiency (Table 3 ). In addition, the absence of the Dxylose and D-fructose catabolic pathways in the entire Shewanella group was also confirmed by their inability to grow on either of these sugars as a carbon and energy source. The only two observed inconsistencies, the inability of $S$. oneidensis to grow on maltodextrin and of S. frigidimarina to grow on mannitol, can be rationalized by genetic rearrangements. Among the genes in the mal locus of $S$. oneidensis disrupted by insertion elements and frameshifts is an already mentioned transporter $\mathrm{GlcP}^{\mathrm{Mal}}$. This observation also provided an explanation of the intrinsic inability of S. oneidensis to grow on Glc, in contrast to many other Shewanella spp, including ANA-3, where the role of this transporter in Glc utilization was experimentally confirmed. It is worth mentioning that our phenotype predictions are largely consistent with the results of earlier growth studies published for a limited set of strains and sugar substrates [13,15,32-35].

Analysis of the distribution of sugar utilization pathways within the Shewanella genus and a comparison with E. coli and other bacteria with different life-styles and natural environments yields interesting observations and conjectures about their adaptive evolution (Fig. 4). A clear distinction between the core and the rare pathways reflects an apparent difference in their evolutionary history. As mentioned above, the former group probably 
existed in a common ancestor of Shewanella spp. Moreover, some of these pathways appear to remain essential in most of the diverse Shewanella habitats as they were lost in only a few rare cases. Thus, the Nag pathway is preserved in 18 and the Grt pathway in 17 of the 19 compared species. This is reminiscent of the distribution of a recently described lactate utilization machinery [8], which is present in all but one species consistent with its proposed role of the main natural supplier of carbon and energy characteristic of the entire Shewanella genus. On the other hand, rare pathways (present in 1-4 species) have more likely originated via lateral gene transfer events, possibly from species sharing the same ecological niche. This may be illustrated by the example of the Gnt utilization pathway that is present in all 4 sequenced strains of S. baltica but not in any other Shewanella spp. A conservation of sequence and genomic arrangement within the Gnt and Rbs gene loci between some closely related Shewanella strains and distant members of Enterobacteriaceae, Pasteurellaceae and Vibrionaceae points to their likely acquisition via a relatively recent lateral gene transfer event (see additional data file 10).

\section{Conclusions}

Overall, the results of this study revealed elements of conservation and variation that appear to be characteristic of the sugar utilization machinery. All reconstructed pathways show a strong tendency to be encoded within compact operons and regulons, and nearly all of the newly identified individual genes are at least distantly homologous (and functionally related) to those previously characterized in other species and included in our original collection of sugar utilization subsystems.

The reconstructed sugar catabolome in Shewanella spp utilizes 170 distinct proteins forming 17 peripheral sugar catabolic pathways and CCM (Table 2, Fig. 1). An apparent "core" subset of peripheral pathways for the utilization of Nag, Grt, Mal and Glc is conserved in $>70 \%$ of compared Shewanella genomes. This level of conservation not only points to their ancestral origin but to an apparent importance of these pathways (and respective sugars) for the physiology of Shewanella spp in various ecosystems. All other peripheral pathways are present in $<50 \%$ of the compared genomes, and among them, 9 pathways are present only in 1-4 species. The latter group seemingly originates from the lateral gene transfer and likely reflects adaptation to specific environmental conditions, which is particularly notable in $S$. frigidimarina and S. pealeana, each containing nonoverlapping sets of 3 "rare" pathways (Fig. 4). Even the most versatile Shewanella species implement only a fraction of the entire pan-Shewanella sugar catabolome reflective of their relatively "lean sugar diet".

\section{Methods}

\section{The subsystems approach and the SEED platform}

Our approach to the reconstruction of sugar catabolic pathways in a selected group of genomes was based on functional gene annotation and prediction using two principal comparative genomics techniques: (i) homology-based methods and (ii) genome context analysis. Both these methods are implemented in the SEED genomic platform http://theseed.uchicago.edu/FIG/ that combines a large and rapidly growing integration of $>700$ complete annotated genomes (mostly bacterial) with advanced tools for comparative analysis, gene annotation, genome context analysis and functional reconstruction based on subsystems technology [7]. New genomes are automatically annotated by the RAST server http://rast.nmpdr.org/, a new generation of subsystem-based genome annotation tools [36]. Subsystems in the SEED provide the framework for further improvement of these annotations and functional predictions. They are sets of functional roles that capture the current knowledge of cellular processes and metabolic pathways including interspecific variation. Each functional role is typically associated with a set of homologous genes that implement this role in specific organisms. In addition to homology-based analysis suggesting at least general class gene functional assignments, genome context analysis provides evidence of functional coupling between genes of known and unknown functions [10]. The most common type of functional coupling evidence comes from the tendency of functionally related genes (e.g., members of the same pathway) to be clustered on the chromosome. Other important types of evidence are domain fusion events, conservation of upstream regulatory sites (i.e., reg-ulons) and co-occurrence profiles of genes across a range of genomes. We used the tools in SEED and other public servers to compute and analyze all types of functional coupling evidence for each gene family in our analysis.

\section{Reconstruction of regulons}

For identification of a candidate regulatory motif for a particular sugar catabolic pathway we started from a training set of potentially co-regulated genes participating in the pathway. Upstream regions of genes from the training set and their orthologs from multiple Shewanella genomes were used as an input for a DNA motif detection algorithm. A simple iterative procedure implemented in the program SignalX (as described previously in [31]) was used for construction of a common transcription factor-binding motif in sets of upstream gene fragments. Each genome encoding the studied transcription factor was scanned with the constructed profile using the GenomeExplorer software [37], and genes 
with candidate regulatory sites in the upstream regions were selected [38]. The threshold for the site search was defined as the lowest score observed in the training set. Among new candidate members of a regulon, only genes having candidate sites conserved in at least two other genomes were retained for further analysis. We also included new candidate regulon members that are functionally related to the reconstructed sugar catabolic pathways. Sequence logos for derived regulatory motifs were drawn using the WebLogo package http://weblogo. berkeley.edu[39]. The details of reconstructed regulons are captured and displayed in the specialized database RegPrecise http://regprecise.lbl.gov[40].

\section{Workflow}

Reconstruction of metabolic and regulatory pathways involved in the carbohydrates utilization was performed for 19 species of the Shewanella genus with completely sequenced genomes uploaded from Genbank and integrated in the SEED genomic platform. The overall workflow is illustrated by Fig. 1. First we performed a survey of all prokaryotic genes known to be involved or potentially involved in utilization of mono- and di-saccharides. A collection of $\sim 480$ FIGfams from 35 SEED sugar metabolic subsystems were classified by their general functional role (i.e. sugar transport, transcriptional regulation, biochemical transformation, and upstream/auxiliary) (see additional data file 1). Each FIGfam comprises a functionally uniform group of orthologous proteins in related organisms. This extensive collection was then used for homology searches against 19 Shewanella genomes resulting in identification of numerous FIGfams potentially implicated in sugar metabolism. Manual curation of the identified Shewanella FIGfams using the SEED and other genomic resources and tools (see below) rejected many of them as well as identified some additional candidate FIGfams based on genome context analysis. As a result of this iterative process we have identified $\sim 170$ FIGfams present in at least one Shewanella genome and tentatively assigned a role in utilization of a paticular sugar substrate (see additional data file 2). The identified FIGfams were used for metabolic reconstruction of Shewanella sugar catabolic pathways using the subsystems-based approach. The refined functional annotations were combined in the aggregated SEED subsystem 'Sugar catabolome in Shewanella species'. Besides SEED, we routinely use other bioinformatic tools and databases featuring: genomes (Genbank), gene annotations (UniProt, IMG), primary literature (PubMed), reactions and pathways (KEGG, BioCyc), conserved domains and motifs (COG, PFAM, ProDom), distant homology searches and alignments (PsiBlast, FFAS, T-Coffee), genome context and occurence profiles (STRING, Microbes on Line), transcriptional regulation
(RegTransBase, RegulonDB), protein localization prediction (TMPRED, SignalP).

\section{Experimental validation of functional predictions Bacterial strains, plasmids, and reagents}

E. coli strains DH5 $\alpha$ (Invitrogen, Carlsbad, CA) and the knockout mutant $\triangle b g l F$ from the KEIO collection (a kind gift from Dr. Mori) [41], were used for complementation analyses. E. coli BL21/DE3 (Gibco-BRL, Rockville, MD) was used for protein overexpression and purification. For expression of genes in E. coli, a pBADTOPO vector containing the arabinose promoter (Invitrogen, Carlsbad, CA) or a pET-derived vector containing the $\mathrm{T} 7$ promoter, $\mathrm{His}_{6}$ tag, and tobacco etch virus-protease cleavage site were used. Enzymes for PCR and DNA manipulations were from New England Biolabs Inc. (Beverly, MA). Plasmid purification kits were from Promega (Madison, WI). PCR purification kits and nickel-nitrilotriacetic acid (Ni-NTA) resin were from QIAGEN Inc. (Valencia, CA). Oligonucleotides for PCR and sequencing were synthesized by Sigma-Genosys (Woodlands, TX). All other chemicals, including sucrose, cellobiose, NADH, ATP, phosphenolpyruvate, lactate dehydrogenase, pyruvate kinase, D-glucose, 2-deoxy-D-glucose, D-mannose, D-galactose, D-allose, $\mathrm{D}$-fructose, $\mathrm{L}$-sorbose, $\mathrm{D}$-tagatose, $\mathrm{D}$-glucosamine, $\mathrm{D}$ mannosamine, D-galactosamine, $\mathrm{N}$-acetylglucosamine, $\mathrm{N}$-acetylmannosamine, $\mathrm{N}$-acetylgalactosamine were purchased from Sigma-Aldrich (St. Louis, MO).

\section{$P C R$ amplification and cloning}

The 2.9-kb fragment from Shewanella frigidimarina NCIMB400, which contains two divergently transcribed genes, scr $T^{I I}$ and $\operatorname{scr} P$ (Sfri_3989 and Sfri_3990, respectively) and the intergenic region, was amplified using the primers: 5'-gcgTTATTTAGCGTCTGCGGTCAACAAATG (forward-1) and 5'-gagTTATCCGCTGTGTTTAGCCAGTAAATC (reverse-1). For cloning the fragment containing $s c r T^{I I}$ only and the intergenic region, the primers of forward-1 and 5'-CGCTATACGATCTACGTAAGTGATCAGCTG were used. For cloning the fragment containing scrP only and the intergenic region, the primers of $5^{\prime}$-CTCATTATCCTTCATATGGTTAACCATAC and reverse- 1 were used.

The 2.7-kb fragment from Shewanella baltica OS155, which contains the $b g l A^{I}$ and $\operatorname{bglT}$ genes (Sbal_0544 and Sbal_0545, repectively), was amplified using the primers: 5'-gaggaataataaATGAAAATATCTTTACCAAAG (forward-2) and 5'-cacTTAGCTGGCTCTATTTAATTCCAG (reverse-2). For cloning Sbal_0544 only, the primers of forward-2 and 5'-gccTTATTTATTATTGTTATTAGTAAAGTGAGC were used. For cloning Sbal_0545 only, the primers of 5'-gaggaataataaATGATTAGCATAAAAGAAAAAATAG and reverse- 2 were used. Nucleotides not present in the original sequence 
are shown in lowercase. PCR amplification was performed using the genomic DNAs of S. frigidimarina NCIMB400 and S. baltica OS155. The PCR fragments as describe above were cloned into the pBAD-TOPO expression vector.

The $g l k^{\mathrm{II}}$ gene from S. baltica OS155 (Sbal_1134) was amplified using the primers: $5^{\prime}$-ggcgcacATGTTACGAATTGGTATCGATCTTG (forward-3) and 5'gcaacgtcgacTTAGCGTCCCCACAACCAAGC (reverse3). Introduced restriction sites (PciI for forward-3 primer and SalI for reverse-3 primer) are shown in boldface. PCR fragment was cloned into the pET-derived expression vector cleaved by NcoI and SalI. Selected clones were confirmed by DNA sequence analysis.

\section{Protein purification}

Recombinant protein of $g^{\mathrm{II}}$ (Sbal_1134) from S. baltica OS155 was overexpressed as N-terminal fusion with a $\mathrm{His}_{6}$ tag in E. coli strain BL21/DE3. Cells were grown on $\mathrm{LB}$ media to $\mathrm{OD}_{600}=0.8$ at $37^{\circ} \mathrm{C}$, induced by $0.2 \mathrm{mM}$ IPTG, and harvested after $12 \mathrm{~h}$ shaking at $20^{\circ} \mathrm{C}$. Protein purification was performed using rapid $\mathrm{Ni}$-NTA agarose minicolumn. Briefly, harvested cells were resuspended in $20 \mathrm{mM}$ HEPES buffer $\mathrm{pH} 7$ containing $100 \mathrm{mM} \mathrm{NaCl}, 0.03 \%$ Brij 35, and $2 \mathrm{mM} \beta$ mercaptoethanol supplemented with $2 \mathrm{mM}$ phenylmethylsulfonyl fluoride and a protease inhibitor cocktail (Sigma-Aldrich). Lysozyme was added to $1 \mathrm{mg} / \mathrm{mL}$, and the cells were lyzed by freezing-thawing followed by sonication. After centrifugation at 18,000 $\mathrm{rpm}$, the Tris- $\mathrm{HCl}$ buffer ( $\mathrm{pH} 8)$ was added to the supernatant (50 mM, final concentration), and it was loaded onto a Ni-NTA agarose column $(0.2 \mathrm{ml})$. After washing with the starting buffer containing $1 \mathrm{M} \mathrm{NaCl}$ and $0.3 \%$ Brij- 35 , bound proteins were eluted with 0.3 $\mathrm{ml}$ of the starting buffer containing $250 \mathrm{mM}$ imidazole. Protein size, expression level, distribution between soluble and insoluble forms, and extent of purification were monitored by SDS-PAGE.

\section{Complementation analyses}

In all complementation analyses, cells were pre-cultured on LB media to exponential growth phase, harvested by centrifugation, and washed for three times with M9 minimal media without any carbon sources. All cultures were started with the same optical density at $600 \mathrm{~nm}$ $\left(\mathrm{OD}_{600 \mathrm{~nm}}=0.03\right)$, and performed at $37^{\circ} \mathrm{C}$ in triplicates in $200 \mu \mathrm{l}$ of the respective media. The cell growth was monitored spectrophotometrically at $600 \mathrm{~nm}$ using a microplate reader (ELx808, BioTek Inc., Winnoski, Vermont).

Recombinant proteins of $S$. frigidimarina NCIMB400 ScrT $^{\text {II }}$ (Sfri_3989) only, ScrP (Sfri_3990) only, and $\mathrm{ScrT}^{\mathrm{II}}$-ScrP were expressed in E. coli $\mathrm{K}-12$ strain DH5 $\alpha$ under the control of endogenous promoter in the intergenic region. The empty $\mathrm{pBAD}-\mathrm{TOPO}$ vector was expressed in the same strain and used as a negative control. The complementation analysis was performed on M9 minimal media supplemented with $50 \mu \mathrm{g} / \mathrm{ml}$ of thiamine and $20 \mathrm{mM}$ of sucrose.

Recombinant proteins of S. baltica OS155 BglA ${ }^{\mathrm{I}}$ (Sbal_0544) only, BglT (Sbal_0545) only, and BglA ${ }^{\mathrm{I}}$ BglT were expressed under the control of arabinose promoter in E. coli $\triangle b g l F$ mutant. The empty pBAD-TOPO vector was expressed in the same strain and used as a negative control. The complementation analysis was performed on M9 minimal media supplemented with $0.15 \%$ of L-arabinose and $20 \mathrm{mM}$ of cellobiose.

\section{In vitro enzyme assays}

Glucokinase activity was assayed by coupling the formation of ADP to the oxidation of NADH to $\mathrm{NAD}^{+}$via pyruvate kinase and lactate dehydrogenase and monitored at $340 \mathrm{~nm}$. Briefly, 0.1-0.2 $\mu \mathrm{g}$ of purified glucokinase was added to $200 \mu \mathrm{L}$ of reaction mixture containing $50 \mathrm{mM}$ Tris buffer (pH 7.5), $10 \mathrm{mM} \mathrm{MgSO}_{4}$, $1.2 \mathrm{mM}$ ATP, $1.2 \mathrm{mM}$ phosphoenolpyruvate, $0.3 \mathrm{mM}$ $\mathrm{NADH}, 1.2 \mathrm{U}$ of pyruvate kinase, $1.2 \mathrm{U}$ of lactate dehydrogenase, and $5 \mathrm{mM} \mathrm{D}$-glucose at $37^{\circ} \mathrm{C}$. No activity was detected in a control experiment, in which an unrelated gene (SO3505) was expressed in the same vector and purified in parallel. The substrate specificities were examined by using the same assay method and exchanging glucose for $5 \mathrm{mM}$ of other potential substrates: 2-deoxyglucose, D-mannose, D-galactose, D-allose, D-fructose, L-sorbose, $N$-acetyl-D-mannosamine, $\mathrm{N}$-acetyl-D-glucosamine, $\mathrm{N}$-acetyl-D-galactosamine, D-galactosamine, D-glucosamine, D-mannosamine. In the coupled assays, the change in NADH absorbance was monitored at $340 \mathrm{~nm}$ using a Beckman DTX-880 multimode microplate reader. An NADH extinction coefficient of $6.22 \mathrm{mM}^{-1} \mathrm{~cm}^{-1}$ was used for rate calculation.

\section{Phenotypic analysis of Shewanella spp}

Total 14 Shewanella strains were tested for their ability to grow on 20 different carbon sources as a sole carbon and energy source. D-/L-lactate mixture was used as a control. Growth conditions and other details of two different experimental techniques used for growth phenotype analysis are provided in additional files 7 and 8.

\section{In-frame deletion mutagenesis}

In-frame deletion mutagenesis of $g l y T$ (SO1771) or nagP (SO3503) was performed using previously published method [42] with minor modifications. Upstream and downstream fragments flanking the target locus were PCR amplified using S. oneidensis MR-1 genomic DNA and fused via overlap extension PCR. The fusion PCR amplicon was ligated into XcmI-digested pDS3.0. The resulting recombinant plasmids were used to transform E. coli $1-2155$ or WM3063 and subsequently transferred to $S$. oneidensis strain MR-1 by conjugation. The primary integrants were selected by plating on LB medium 
containing $7.5 \mu \mathrm{g} / \mathrm{ml}$ gentamycin. The screening for a second round of homologous recombination to remove the plasmid sequence was accomplished by counterselection on LB medium with $5 \%$ sucrose. Colonies growing in the presence of sucrose were screened for sensitivity to gentamycin $(7.5 \mu \mathrm{g} / \mathrm{ml})$ and then screened for deletion of the gene of interest using PCR. The resulting PCR amplicon was then used as the template for DNA sequencing of the deleted and flanking regions involved in the recombination events (ACGT, Inc. Wheeling, IL). In order to validate that the observed phenotype could be attributed to the targeted deletion, complementing plasmids were constructed by inserting appropriate genes downstream to the lac promoter encoded in PBBR1MCS-5.

\section{Additional material}

\section{Additional file 1: Collection of protein families involved in carbohydrate utilization in bacteria.}

Additional file 2: Summary on distribution of carbohydrate utlization genes in Shewanella spp.

Additional file 3: Complete distribution of sugar catabolome genes in Shewanella genomes.

Additional file 4: Description of rare sugar utilization pathways in Shewanella.

Additional file 5: Predicted novel functional roles for isofunctional protein families from Shewanella sugar catabolome.

Additional file 6: Experimental verification of novel enzymes, transporters, and regulators involved in sugar utilization in Shewanella. A. Phenotypic characterization of glc ${ }^{\text {Mal }}$ (Shewana3_2310) for its involvement in glucose utilization in Shewanella sp. ANA-3; B. Phenotypic characterization of nagP (SO3503) for its involvement in $\mathrm{N}$ acetylglucosamine (Nag) utilization in Shewanella oneidensis MR-1; C. Phenotypic characterization of grtP (SO1771) for its involvement in Dglycerate; D. Complementation of the E. coli cellobiose utilization by the bglA-bglT (Sbal_1133-1132) genes from S. baltica OS155; E. Substrate specificity of Shewanella baltica OS155 GIk" (Sbal_1134) kinase; F. Growth of E. coli DH5a strain (Scr-) containing heterologously expressed sucrose utilization genes scrTll-scrP (Sfri_3989-3990) from S. frigidimarina.

Additional file 7: Growth phenotypes of Shewanella on various carbon sources determined by manual assay.

Additional file 8: Growth phenotypes of Shewanella on various carbon sources determined by microplate assay using Bioscreen $C$ MBR system.

Additional file 9: Comparison of reconstructed sugar utilization pathways in Shewanella and Enterobacteria. A. N-acetylglucosamine (Nag) and chitin utilization pathways; B. D-glycerate (Grt) and glucarate/ galactarate utilization pathways; C. $\beta$-glucoside (Bgl) utilization pathway; D. Sucrose (Scr) utilization pathway; E. L-arabinose (Ara) and arabinosides utilization pathways.

Additional file 10: The gluconate (A) and ribose (B) utilization gene loci in some closely related Shewanella strains and other $\gamma$ proteobacteria.

\section{List of abbreviations}

PTS: phosphotransferase system; FIGFAMS: isofunctional protein families in the SEED database; CCN: central carbon metabolism; ED pathway: EntnerDoudoroff pathway; PP pathway: pentose phosphate pathway; TBDT: TonBdependent outer membrane transporter; Aga: $\mathrm{N}$-acetylgalactosamine; Ara: L- arabinose; Bgl: $\beta$-glucoside (cellobiose); Gal: D-galactose; Glc: D-glucose; Gnt: D-gluconate; Grt: D-glycerate; Mal: maltodextrin; Man: mannosides; Mtl: mannitol; Nag: N-acetylglucosamine; Nan: sialic acids; Rbs: D-ribose; Scr: sucrose; Tre: trehalose; Xlt: xylitol.

\section{Authors' contributions}

$D A R, C Y$, and $A O$ conceived and supervised the research, and wrote the manuscript. DAR performed comparative genomic analysis to infer novel metabolic pathways and regulons. RO and OPZ performed computational similarity searches, gene annotation and subsystem encoding in the SEED database. $C Y, X L$, and IAR carried out genetic complementation experiments. MFR and SR produced targeted gene knockout strains in Shewanella. YW, AYO, and SR performed phenotypic characterization of Shewanella strains. JKF and KHN contributed to the development of the manuscript and design of the study. All authors read and approved the final manuscript.

\section{Acknowledgements}

We are grateful to Pavel Novichkov (LBNL) for help with regulon analysis and presentation in RegPrecise database. This research was supported by the U.S. Department of Energy (DOE) Office of Biological and Environmental Research under the Genomics:GTL Program via the Shewanella Federation consortium and the Microbial Genome Program (MGP). Pacific Northwest National Laboratory is operated for the DOE by Battelle Memorial Institute under Contract DE-AC05-76RLO 1830. D.A.R. was partially supported by National Science Foundation (DBI-0850546), Russian Foundation for Basic Research (10-04-01768), Russian Academy of Sciences program 'Molecular and Cellular Biology' and Russian President's grant for young scientists (MK422.2009.4). C. Y. was supported by National Science Foundation of China (30970035) and One-hundred-Talented-People program of Chinese Academy of Sciences (KSCX2-YW-G-029).

\section{Author details}

${ }^{1}$ Burnham Institute for Medical Research, La Jolla, California 92037, USA. ${ }^{2}$ Institute for Information Transmission Problems, Russian Academy of Sciences, Moscow 127994, Russia. ${ }^{3}$ Key Laboratory of Synthetic Biology, Institute of Plant Physiology and Ecology, Shanghai Institutes for Biological Sciences, Chinese Academy of Sciences, Shanghai 200032, China. ${ }^{4}$ Department of Earth Sciences, University of Southern California, Los Angeles, California 90089, USA. ${ }^{5}$ Fellowship for Interpretation of Genomes, Burr Ridge, Illinois 60527, USA. ${ }^{6}$ Biological Sciences Division, Pacific Northwest National Laboratory, Richland, Washington 99352, USA. ${ }^{7} \mathrm{~J}$. Craig Venter Institute, San Diego, California 92121, USA.

Received: 24 June 2010 Accepted: 13 September 2010 Published: 13 September 2010

\section{References}

1. Fredrickson JK, Romine MF, Beliaev AS, Auchtung JM, Driscoll ME, Gardner TS, Nealson KH, Osterman AL, Pinchuk G, Reed JL, Rodionov DA, Rodrigues JL, Saffarini DA, Serres MH, Spormann AM, Zhulin IB, Tiedje JM: Towards environmental systems biology of Shewanella. Nat Rev Microbiol 2008, 6:592-603.

2. Hau HH, Gralnick JA: Ecology and biotechnology of the genus Shewanella. Annu Rev Microbiol 2007, 61:237-258.

3. Serres MH, Riley M: Genomic analysis of carbon source metabolism of Shewanella oneidensis MR-1: Predictions versus experiments. J Bacteriol 2006, 188:4601-4609.

4. Pinchuk GE, Ammons C, Culley DE, Li SM, McLean JS, Romine MF, Nealson KH, Fredrickson JK, Beliaev AS: Utilization of DNA as a sole source of phosphorus, carbon, and energy by Shewanella spp.: ecological and physiological implications for dissimilatory metal reduction. Appl Environ Microbiol 2008, 74:1198-1208.

5. Driscoll ME, Romine MF, Juhn FS, Serres MH, McCue LA, Beliaev AS, Fredrickson JK, Gardner TS: Identification of diverse carbon utilization pathways in Shewanella oneidensis MR-1 via expression profiling. Genome Inform 2007, 18:287-298.

6. Osterman A, Overbeek R: Missing genes in metabolic pathways: a comparative genomics approach. Curr Opin Chem Biol 2003, 7:238-251.

7. Overbeek R, Begley T, Butler RM, Choudhuri JV, Chuang HY, Cohoon M, de Crecy-Lagard V, Diaz N, Disz T, Edwards R, Fonstein M, Frank ED, Gerdes S, Glass EM, Goesmann A, Hanson A, Iwata-Reuyl D, Jensen R, Jamshidi N, 
Krause L, Kubal M, Larsen N, Linke B, McHardy AC, Meyer F, Neuweger H, Olsen G, Olson R, Osterman A, Portnoy V, Pusch GD, Rodionov DA, Ruckert C, Steiner J, Stevens R, Thiele I, Vassieva O, Ye Y, Zagnitko O, Vonstein $\mathrm{V}$ : The subsystems approach to genome annotation and its use in the project to annotate 1000 genomes. Nucleic Acids Res 2005, 33:5691-5702.

8. Pinchuk GE, Rodionov DA, Yang C, Li X, Osterman AL, Dervyn E, Geydebrekht OV, Reed SB, Romine MF, Collart FR, Scott JH, Fredrickson JK, Beliaev AS: Genomic reconstruction of Shewanella oneidensis MR-1 metabolism reveals a previously uncharacterized machinery for lactate utilization. Proc Natl Acad Sci USA 2009, 106:2874-2879.

9. Yang C, Rodionov DA, Li X, Laikova ON, Gelfand MS, Zagnitko OP, Romine MF, Obraztsova AY, Nealson KH, Osterman AL: Comparative genomics and experimental characterization of $\mathrm{N}$-acetylglucosamine utilization pathway of Shewanella oneidensis. J Biol Chem 2006, 281:29872-29885.

10. Overbeek R, Fonstein M, D'Souza M, Pusch GD, Maltsev N: The use of gene clusters to infer functional coupling. Proc Natl Acad Sci USA 1999, 96:2896-2901.

11. Meyer F, Overbeek R, Rodriguez A: FIGfams: yet another set of protein families. Nucleic Acids Res 2009, 37:6643-6654.

12. Scott JH, Nealson KH: A biochemical study of the intermediary carbon metabolism of Shewanella putrefaciens. J Bacteriol 1994, 176:3408-3411.

13. Bowman JP, McCammon SA, Nichols DS, Skerratt JH, Rea SM, Nichols PD, McMeekin TA: Shewanella gelidimarina sp. nov. and Shewanella frigidimarina sp. nov., novel Antarctic species with the ability to produce eicosapentaenoic acid (20:5 omega 3) and grow anaerobically by dissimilatory Fe(III) reduction. Int J Syst Bacteriol 1997, 47:1040-1047.

14. Fonnesbech Vogel B, Venkateswaran K, Satomi M, Gram L: Identification of Shewanella baltica as the most important $\mathrm{H} 2 \mathrm{~S}$-producing species during iced storage of Danish marine fish. Appl Environ Microbiol 2005, 71:6689-6697.

15. Leonardo MR, Moser DP, Barbieri E, Brantner CA, MacGregor BJ, Paster BJ, Stackebrandt E, Nealson KH: Shewanella pealeana sp. nov., a member of the microbial community associated with the accessory nidamental gland of the squid Loligo pealei. Int J Syst Bacteriol 1999, 49:1341-1351.

16. Essenberg RC, Candler C, Nida SK: Brucella abortus strain 2308 putative glucose and galactose transporter gene: cloning and characterization. Microbiology 1997, 143:1549-1555.

17. Titgemeyer F, Reizer J, Reizer A, Saier MH Jr: Evolutionary relationships between sugar kinases and transcriptional repressors in bacteria. Microbiology 1994, 140:2349-2354.

18. Romine MF, Carlson TS, Norbeck AD, McCue LA, Lipton MS: Identification of mobile elements and pseudogenes in the Shewanella oneidensis MR-1 genome. Appl Environ Microbiol 2008, 74:3257-3265.

19. Roehl RA, Vinopal RT: Lack of glucose phosphotransferase function in phosphofructokinase mutants of Escherichia coli. J Bacteriol 1976, 126:852-860

20. Monterrubio R, Baldoma L, Obradors N, Aguilar J, Badia J: A common regulator for the operons encoding the enzymes involved in $D$ galactarate, D-glucarate, and D-glycerate utilization in Escherichia coli. $J$ Bacteriol 2000, 182:2672-2674

21. Reizer J, Reizer A, Saier MH Jr: The cellobiose permease of Escherichia coli consists of three proteins and is homologous to the lactose permease of Staphylococcus aureus. Res Microbiol 1990, 141:1061-1067.

22. Laikova ON, Mironov AA, Gelfand MS: Computational analysis of the transcriptional regulation of pentose utilization systems in the gamma subdivision of Proteobacteria. FEMS Microbiol Lett 2001, 205:315-322.

23. Schauer K, Rodionov DA, de Reuse H: New substrates for TonB-dependent transport: do we only see the 'tip of the iceberg'? Trends Biochem Sci 2008, 33:330-338.

24. Reid SJ, Abratt VR: Sucrose utilisation in bacteria: genetic organisation and regulation. Appl Microbiol Biotechnol 2005, 67:312-321.

25. Trindade Ml, Abratt VR, Reid SJ: Induction of sucrose utilization genes from Bifidobacterium lactis by sucrose and raffinose. Appl Environ Microbiol 2003, 69:24-32.

26. Andersen C, Rak B, Benz R: The gene bglH present in the bgl operon of Escherichia coli, responsible for uptake and fermentation of betaglucosides encodes for a carbohydrate-specific outer membrane porin. Mol Microbiol 1999, 31:499-510.
27. Boos W, Shuman H: Maltose/maltodextrin system of Escherichia coli: transport, metabolism, and regulation. Microbiol Mol Biol Rev 1998, 62:204-229.

28. Watanabe S, Shimada N, Tajima K, Kodaki T, Makino K: Identification and characterization of L-arabonate dehydratase, L-2-keto-3-deoxyarabonate dehydratase, and L-arabinolactonase involved in an alternative pathway of L-arabinose metabolism. Novel evolutionary insight into sugar metabolism. J Biol Chem 2006, 281:33521-33536.

29. Omelchenko MV, Galperin MY, Wolf YI, Koonin EV: Non-homologous isofunctional enzymes: a systematic analysis of alternative solutions in enzyme evolution. Biol Direct 2010, 5:31.

30. Tchieu JH, Norris V, Edwards JS, Saier MH Jr: The complete phosphotranferase system in Escherichia coli. J Mol Microbiol Biotechnol 2001, 3:329-346.

31. Rodionov DA: Comparative genomic reconstruction of transcriptional regulatory networks in bacteria. Chem Rev 2007, 107:3467-3497.

32. Brettar I, Christen R, Hofle MG: Shewanella denitrificans sp. nov., a vigorously denitrifying bacterium isolated from the oxic-anoxic interface of the Gotland Deep in the central Baltic Sea. Int I Syst Evol Microbiol 2002, 52:2211-2217.

33. Zhao JS, Manno D, Beaulieu C, Paquet L, Hawari J: Shewanella sediminis sp. nov., a novel Na+-requiring and hexahydro-1,3,5-trinitro-1,3,5triazine-degrading bacterium from marine sediment. Int I Syst Evol Microbiol 2005, 55:1511-1520.

34. Zhao JS, Manno D, Leggiadro C, O'Neil D, Hawari J: Shewanella halifaxensis sp. nov., a novel obligately respiratory and denitrifying psychrophile. Int J Syst Evol Microbiol 2006, 56:205-212.

35. Ziemke F, Hofle MG, Lalucat J, Rossello-Mora R: Reclassification of Shewanella putrefaciens Owen's genomic group II as Shewanella baltica sp. nov. Int J Syst Bacteriol 1998, 48(Pt 1):179-186.

36. Aziz RK, Bartels D, Best AA, DeJongh M, Disz T, Edwards RA, Formsma K, Gerdes S, Glass EM, Kubal M, Meyer F, Olsen GJ, Olson R, Osterman AL, Overbeek RA, McNeil LK, Paarmann D, Paczian T, Parrello B, Pusch GD, Reich C, Stevens R, Vassieva O, Vonstein V, Wilke A, Zagnitko O: The RAST Server: rapid annotations using subsystems technology. BMC Genomics 2008, 9:75.

37. Mironov AA, Vinokurova NP, Gel'fand MS: [Software for analyzing bacterial genomes]. Mol Biol (Mosk) 2000, 34:253-262.

38. Gelfand MS, Koonin EV, Mironov AA: Prediction of transcription regulatory sites in Archaea by a comparative genomic approach. Nucleic Acids Res 2000, 28:695-705.

39. Crooks GE, Hon G, Chandonia JM, Brenner SE: WebLogo: a sequence logo generator. Genome Res 2004, 14:1188-1190.

40. Novichkov PS, Laikova ON, Novichkova ES, Gelfand MS, Arkin AP, Dubchak I, Rodionov DA: RegPrecise: a database of curated genomic inferences of transcriptional regulatory interactions in prokaryotes. Nucleic Acids Res 2010, 38:D111-118.

41. Baba T, Ara T, Hasegawa M, Takai Y, Okumura Y, Baba M, Datsenko KA, Tomita M, Wanner BL, Mori H: Construction of Escherichia coli K-12 inframe, single-gene knockout mutants: the Keio collection. Mol Syst Biol 2006, 2:2006.0008.

42. Wan XF, Verberkmoes NC, McCue LA, Stanek D, Connelly H, Hauser L, Wu L, Liu X, Yan T, Leaphart A, Hettich RL, Zhou J, Thompson DK: Transcriptomic and proteomic characterization of the Fur modulon in the metal-reducing bacterium Shewanella oneidensis. Journal of Bacteriology 2004, 186:8385-8400.

doi:10.1186/1471-2164-11-494

Cite this article as: Rodionov et al:: Genomic encyclopedia of sugar utilization pathways in the Shewanella genus. BMC Genomics 2010 11:494. 